Ann. Abeille, Ig63, 6 (I), 5-33

\title{
SUR QUELQUES COMPOSÉS DE LA GELÉE ROYALE ET DES LARVES DE REINES D’ABEILLES
}

\author{
Daria BOGDANOVSKY \\ Institut de Chimie des Substances naturelles, \\ Service du Professour E. Lederer, Gif-sur-Yostle (Seine-el-Oise)
}

\section{SOMMAIRE,}

Dans le présent travail, nous décrivons la fraction lipidique des larves de reines d'abeilles, et comparons la composition de cette fraction avec celle des lipides de la gelée royale.

Dans une première partie, nous avons recherché, d'une part l'acide hydroxy-Io décène-2 trans oïque, acide présent dans la gelée royale et doué de propriétés biologiques importantes, et d'autre part les produits du métabolisme de cet acide.

Dans une seconde partie nous avons examiné les autres acides, en particulier les acides dicarboxyliques.

Enfin nous avons étudié le problème de la nutrition en stérols des larves de reines.

\section{IN'TRODUCTION}

La gelée royale a fait 1'objet, au cours des dernières années, de nombreuses études chimiques et biologiques. La bibliographie concernant cette substance est relativement abondante, par contre la bibliographie sur les larves de reines d'abeilles est inexistante.

\section{I. - GeítéE ROYAIE}

La gelée royale est une sécrétion des glandes hypopharyngiennes des abeilles ouvrières. Cette sécrétion se distingue des sécrétions hypopharyngiennes des reines d'abeilles, à la fois par sa composition et par ses propriétés biologiques. Elle est fournie aux jeunes larves durant les premiers jours de leur vie. Elle constitue la nourriture unique des larves de reines. Les larves d'ouvrières reçoivent en plus du pollen, dès le troisième jour de leur vie. Chaque cellule royale peut contenir de 200 à $300 \mathrm{mg}$ de gelée royale.

La gelée royale se présente sous forme d'un lait blanc visqueux. Flle contient, d'après BUTENANDT (I959), 60 p. Ioo d'eau, 30 p. Ioo d'hydrosoluble dont I 5 p. Ioo de sucre inverti, et enfin Io p. Ioo d'éthérosoluble. 


\section{A) Propriétés biologiques}

De nombreux travaux ont été effectués sur les propriétés biologiques de la gelée royale. Chauvin (I959) a résumé ses propriétés thérapeutiques et son emploi dans les convalescences. La gelée royale est particulièrement tiche en vitamines.

Les travaux de Townsend, MorGan et HazlettT (I959) (I960) ont montré que la gelée royale inhibe le développement de la leucémie et de certaines variétés de tumeurs chez la Souris. Cette activité est due en partie à l'acide hydroxy-Io décène-2 trans oïque, isolé par TownsEnd et Lucas (r940) et identifié par BuTENAND'T et REMBOId (I957).

A cette action, doit s'ajouter celle des acides dicarboxyliques, dont l'activité antileucémique a également été étudiée par Morgan, Tolnai et Townsknd (I960).

La gelée royale possède, in vitro, une activité antibiotique, qui serait également due à 1'acide hydroxy-Io décène-2 trans oïque. Cette activité a été étudiée par Bı,um, Novak et TABER (I959).

\section{B) Acides de la gelée royale}

a) Acide hydroxy-Io décène-2 trans ö̈que.

'TOWNSEND et LUCAS (I940) ont isolé de la gelée royale, par extraction à l'éther un acide $\mathrm{C}_{10} \mathrm{H}_{18} \mathrm{O}_{3}$. La structure de cet acide a été déterminée par BUTENAxD'T et REMBOLD (I957).

La réduction cle l'acide hydroxy-Io décène-2 trans oïque par l'hydrure double de lithium et d'aluminium, conduit au décane-diol I, Io. La réduction par 1'hydrogène en présence de platine, donne l'acide hydroxy-Io décanoïque. L'oxydation de ces deux alcools conduit à l'acide sébacique. L'acide sébacique lui-même peut être obtenu, après oxydation ménagée en acide décène- 2 dioïque, suivie d'hydrogénation de cet acide.

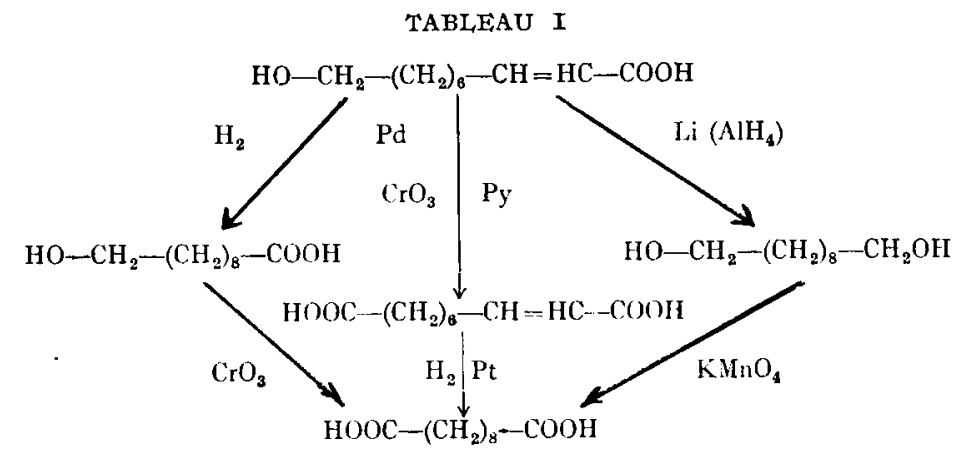

Ainsi que nous l'avons vu, les propriétés biologiques de cet acide sont responsables, en partie, de celles de la gelée royale.

L'activité antitumorale étudiée par 'Townsend et al. (I959) (I960) a été de nouveau étudiée par RoBIsson (I960). Selon cet auteur, il y aurait formation d'un poly-ester.

$$
\begin{aligned}
n\left[\mathrm{HO}-\left(\mathrm{CH}_{2}\right)_{7}-\mathrm{CH}=\mathrm{CH}-\mathrm{COOH}\right] & \rightarrow \mathrm{HO}-\left(\mathrm{CH}_{2}\right)_{7}-\mathrm{CH}=\mathrm{CH}-\mathrm{CO}\left[\mathrm{O}\left(\mathrm{CH}_{2}\right)_{7}-\mathrm{CH}\right. \\
= & \mathrm{CH}-\mathrm{CO}]^{n-2}-\mathrm{O}-\left(\mathrm{CH}_{2}\right)_{7}-\mathrm{CH}=\mathrm{CH}-\mathrm{COOH} .
\end{aligned}
$$


Ces grosses molécules, ainsi formées pouvant adhérer à la surface des cellules, cellesci sont entourées et freinées dans leur multiplication.

La synthèse de cet acide ainsi que celle de son isomère cis, ont été réalisées par Robinson et $a$. . (I960, $a, b)$. Les synthèses de 1'acide hydroxy-Io décène-2- trans oïque passent par l'intermédiaire de l'acétate d'hydroxy-8 octanal, dont l'obtention par 3 voies différentes est décrite. Ce composé est ensuite condensé sur l'acide malonique dans la pyridine, pour conduite à l'acide acétoxy-ı décène-2 trans oïque. Une autre voie de synthèse, à partir de l'acide undécylènique, est proposée par ces mêmes auteurs.

Enfin une modification de la première méthode a été proposée par RAPHAEL, (cité par RoBINSON) (I960).

L'acide hydroxy-Io décène-2 trans oïque, est une production spécifique des glandes mandibulaires des abeilles ouvrières. Les reines d'abeilles produisent une sécrétion très différente. L'acide céto-9 décène-2 trans oïque (Substance royale) a pu être isolé et identifié par CALLOW et JoHNSTON (Ig60) et par BARBIER et al. (Ig60, $a, c, d)$.

$$
\mathrm{H}_{3} \mathrm{C}-\mathrm{CO}-\left(\mathrm{CH}_{2}\right)_{5}-\mathrm{CH}=\mathrm{CH}-\mathrm{COOH}
$$

On sait que les sécrétions mandibulaires des reines d'abeilles jouent un rôle très important; elles sont attractives vis-à-vis des abeilles ouvrières et celles-ci venant lécher leur reine ingèrent ces sécrétions mandibulaires. Il s'ensuit :

- d'une part, ainsi que l'ont montré BUTLER (I957) et PAIN (I957) une inhition de leurs ovaires;

- d'autre part, une inhibition de la construction des cellules royales. Ce phénomène a été étudié par BuTLER (I957) (I959).

\section{b) Autres acides de la gelée royale.}

La présence de l'acide hydroxy-Io décène-2 trans oïque dans la gelée royale permettait de prévoir celle d'une série d'acides pouvant en dériver. L'acide sébacique, signalé par BROWN et FREURE (I959), semble avoir été le premier acide dicarboxylique mis en évidence dans la gelée royale.

$$
\mathrm{HOOC}-\left(\mathrm{CH}_{2}\right)_{8}-\mathrm{COOH}
$$

La présence d'acides dicarboxyliques dans la nature est assez rare.

BROWN et FREURE (I959) signalent également la présence dans la gelée royale, de 1'acide décène-2 trans dioïque. La synthèse de cet homologue de l'acide traumatique a été décrite par ENGLISH (I94I).

$$
\mathrm{HOOC}-\left(\mathrm{CH}_{2}\right)_{6}-\mathrm{CH}=\mathrm{CH}-\mathrm{COOH}
$$

BROWN, FELAUER et FREURE (I96I), ont isolé l'acide hydroxy-Io décanoïque, dont la synthèse à partir de l'acide undécylénique était déjà connue d'après les travaux de BENTON et KIESS (I960).

$$
\mathrm{HO}-\mathrm{CH}_{2}-\left(\mathrm{CH}_{2}\right)_{8}-\mathrm{COOH}
$$

Dans le même travail, les auteurs signalent la présence de l'acide p-hydroxy benzoïque.

$$
\mathrm{HO}-\mathrm{C}_{6} \mathrm{H}_{4}-\mathrm{COOH}
$$


Selon les recherches récentes de BROWN et FELAUER (I96I) l'acide hydroxy-9 décène-2 trans oïque serait également présent dans la gelée royale. Cependant son isolement n'a pas été décrit. Si cette présence était confirmée il serait possible de l'envisager comme précurseur de la substance royale.

TABLEAU 2

Acides de la gelée royale

\begin{tabular}{|c|c|c|c|}
\hline Substances & Formules & P. F. & $\%$ \\
\hline 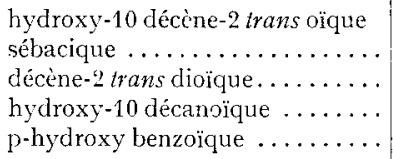 & $\begin{array}{l}\mathrm{HO} \mathrm{CH}_{2}-\left(\mathrm{CH}_{2}\right)_{6} \ldots \mathrm{CH}=\mathrm{CH}-\mathrm{COOH} \\
\mathrm{HOOC}-\left(\mathrm{CH}_{2}\right)_{8}-\mathrm{COOH} \\
\mathrm{IOOC} \cdot\left(\mathrm{CH}_{2}\right)_{6} \mathrm{CH}=\mathrm{HC}-\mathrm{COOH} \\
\mathrm{HO}-\mathrm{CH}_{2}-\left(\mathrm{CH}_{2}\right)_{8}-\mathrm{COOH} \\
\mathrm{HO}-\mathrm{C}_{6} \mathrm{H}_{4}-\mathrm{COOH}\end{array}$ & $\begin{array}{c}65^{\circ} \\
133^{\circ} \\
160-70^{\circ} \\
72^{\circ} \\
214^{\circ}\end{array}$ & $\begin{array}{l}3 \% \\
0,02 \% \\
0,03 \% \\
0,5 \% \\
0,003 \%\end{array}$ \\
\hline
\end{tabular}

\section{C) Vitamines}

Les différences morphologiques provoquées chez les larves d'abeilles, par la présence de quantités importantes (cas des reines) ou limitées (cas des ouvrières) de gelée royale, ont fait penser à BUTENANDT (I959) que la tenew en vitamines pouvait avoir un rôle particulier.

Nous reportons dans le tableau 3 les pourcentages des vitamines, déterminés dans la gelée royale par ButENANDT et ses collaborateurs.

TABLEAU 3

Vitamines de la gelée royale

\begin{tabular}{|c|c|}
\hline Vitamines & $\begin{array}{l}\text { Gelée royale donnée aux larves } \\
\text { de reines, en } \gamma / g \text { (en poids de } \\
\text { substance fraîche) }\end{array}$ \\
\hline Vitamine $B_{1} \ldots \ldots \ldots \ldots$ & $1,2-18$ \\
\hline Vitamine $B_{2} \ldots \ldots \ldots \ldots$ & $6-28$ \\
\hline Pyridoxine... & $2,2-50$ \\
\hline Acide nicotinique $\ldots \ldots \ldots \ldots \ldots \ldots$ & $48-125$ \\
\hline Biotine $\ldots \ldots \ldots \ldots \ldots \ldots$ & $1,6-4,1$ \\
\hline Acide folique $\ldots \ldots \ldots \ldots \ldots \ldots \ldots$ & $0,16-0,5$ \\
\hline Acide pantothénique...$\ldots \ldots \ldots \ldots$ & $110-320$ \\
\hline Inositol $\ldots \ldots \ldots \ldots \ldots \ldots \ldots \ldots$ & $\overline{78-150}$ \\
\hline
\end{tabular}

La teneut en acide pantothénique est élevée, et PEARSON et BURGIN (I94I) ont montré qu'elle est plus élevée dans la gelée royale qui est distribuée aux larves de reines, que dans celle distribuée aux larves d'ouvrières. Ce fait avait d'abord laissé penser que les quantités d'acide pantothénique étaient déterminantes pour l'induction morphologique.

GARDNER (I948) ayant étudié 1'action de l'acide pantothénique sur les drosophiles a trouvé qu'il augmentait considérablement la durée de leur vie. Une action 
semblable sur les reines est possible; elles vivent en effet plusieurs années, alors que la durée de vie d'une ouvrière dépasse rarement un mois.

L,e fait que l'activité de la gelée royale sur la morphologie des larves soit due au contenu vitaminique n'est pas encore démontré.

\section{I) Bioptérine}

Butenandt et REMBOLD (I958) ont, par chromatographie sur papier, mis en évidence dans la gelée royale, l'amino-2 hydroxy-4 (dihydroxy-I,2 propyl)-6 ptéridine ou bioptérine.

Cette substance présente une tache bleue en U. V. et ces auteurs ont réussi à en isoler $4,7 \mathrm{mg}$ à partir de $500 \mathrm{~g}$ de gelée royale.

La bioptérine avait été précédemment isolée de l'urine humaine par PATTERSON (1955), des drosophiles par ForRest et Mitchell (I955) et par Viscontini (I955). Elle serait également présente d'après Viscoxtrivi (I955 b) (I956) dans les mites et dans les écrevisses.

La synthèse de la bioptérine a été décrite par PATTERsox (I956).

Ce composé joue certainement un rôle biologique important car, ainsi que l'a montré Bu'TENANDT (I959), s'il est possible de le mettre en évidence dans les larves de reines, il est impossible de le détecter dans les larves d'ouvrières.

\section{II. - UTILISATION IJES STĹROLS PAR LES INSECTES}

L,es travaux de CraRk et Bloch (I959 $a, b, c$ ) ont montré que les insectes ne peuvent vivre en absence de stérols. Selon Lipke et FraEnkEL (I956), ce phénomène ne connaît pas d'exception. D'autre part, il semble d'après les travaux de $C_{L A R K}$ et $\mathrm{BI}_{4} \mathrm{OCH}(\mathrm{I} 959$, a) que les insectes soient incapables d'effectuer la biosynthèse des stérols. De très petites quantités de stérols suffisent à leur survie ; ce besoin possède apparemment un caractère analogue aux besoins en vitamines des animaux supérieurs. Les insectes doivent donc absolument trouver dans leur nourriture les stérols nécessaires à leur développenent. 'Tous les stérols ne peuvent remplir ce rôle pour un insecte donné. Les insectes carnivores sont incapables de vivre avec des stérols végétaux (sitostérol, stigmastérol...) comme unique source de stérols.

Le rôle biologique précis des stérols chez l'insecte est jusqu'à présent inconnu. Des travaux récents de KoDICEK et LEvinson (I960) montrent que les phytostérols sont dégradés par oxydation de la ramification en $\mathrm{C}_{24}$.

Des recherches effectuées par Moxroe (I960) avec la mouche domestique, indiquent que le cholestérol joue un rôle dans la reproduction. La survie des adultes et même la ponte, ne sont pas affectés par une diminution de la quantité de stérols dans un régime alimentaire synthétique. Mais l'éclosion des cufs peut se trouver réduite de 80 p. roo dans certains cas. Si ce régime déficient est fourni aux larves, seulement $25 \mathrm{p}$. Ioo arrivent à se métamorphoser.

Il est par ailleurs intéressant de constater, avec les travaux de CLARK et Br,och (I959, a), que les stérols indispensables ne peuvent être remplacés ni par l'acide mévalonique, ou le squalène, ni par le lanostérol ou le diméthyl-4,4' cholestérol; ce qui indique que la voie de biosynthèse des stérols est bloquée de multiples façons. 
(Ces résultats concernant le coléoptère Dermestes vulpinus devront être étendus à d'autres espèces, afin de rendre cette constatation plus générale.)

Du point de vue chimique et du point de vue biologique les besoins et 1'utilisation des stérols par les insectes, sont encore des problèmes non résolus, et dont l'intérêt pour la compréhension de la biologie générale de ces animaux, est vraisemblablement capital.

BARBIER et SchINDLER (I959) ont isolé des abeilles le méthylène-24 cholestérol, stérol principal du pollen; il est remarquable que ce composé n'ait été jusqu'alors isolé que des mollusques par IdLER et FAGERI,UNd (I955). Selon CLARK et Bloch $(I 959, c)$ le méthylène-24 cholestérol est directement utilisable par les insectes phytophages au même titre que le sitostérol.

\section{ÉTUDF SUR LES LARVES DE REINES E'T LA GELÉE ROYALE}

Nous avons eu à notre disposition de grandes quantités de larves de reines (de l'ordre du kg) ce qui représente entre dix et trente mille larves ; nous avons pensé qu'il pouvait être intéressant d'effectuer des recherches sur ce matériel. Ces larves possédant un intestin imperforé, représentent de véritables "sacs à gelée royale ". Nous avons donc entrepris l'étude des lipides de larves de reines, dans les buts suivants : rechercher les constituants de la gelée royale, déterminer les produits de leur métabolisme, et enfin essayer de découvrir de nouvelles substances.

\section{PIAN GÉNÉRAI DE FRACTIONNEMENT DES LARVES DE REINES}

I $\mathrm{kg}$ de larves de reines d'abeilles a été broyé, en suspension dans l'éthanol, et la bouillie ainsi obtenue a été séchée sur plaques d'aluminium, en étuve à $90^{\circ}$, jusqu'à dessiccation complète.

\section{A) Extraction par l'éther}

Une extraction de la poudre résiduelle par l'éther a conduit à I Io g de graisses.

\section{B) Extraction par l'acétone}

Les IIo $\mathrm{g}$ de graisses ont été dissous dans environ $500 \mathrm{ml}$ d'acétone. A froid, on obtient un résidu peu important. Après ébullition à reflux pendant $\mathrm{I} 5 \mathrm{mn}$, l'insoluble disparaît. Il n'est donc pas possible d'isoler de ces lipides une fraction de phosphatides.

\section{C) Séparation par le carbonate de sodium}

Après évaporation de l'acétone, les Iro g de graisses ont été séparés en parties neutres et acides. Pour cela ces I ro g de graisses ont été dissous dans $200 \mathrm{ml}$ d'éther, 
et agités en présence de $200 \mathrm{ml}$ de carbonate de sodium $2 \mathrm{~N}$. On sépare les couches éthérées des couches aqueuses. On effectue trois extractions de cette manière, et on obtient :

a) les extraits aqueux, qui, après acidification par $\mathrm{HCl} 5 \mathrm{~N}$, et extraction à l'éther (l'éther étant lavé 3 fois à l'eau et séché sur sulfate de soude), fournissent $28,8 \mathrm{~g}$ d'acides libres.

b) les extraits éthérés qui, après lavage à l'eau et séchage, fournissent $78,8 \mathrm{~g}$ d'une fraction contenant les neutres et les acides liés.

\section{Saponification de cette fraction.}

Ces $78,8 \mathrm{~g}$ ont été dissous dans $200 \mathrm{ml}$ de benzène, et additionnés de $250 \mathrm{ml}$ de potasse méthanolique $4 \mathrm{~N}$, et 1'ensemble a été chauffé à ébullition pendant 5 heures.

Après refroidissement et addition d'eau, on extrait à l'éther. On obtient ainsi :

- 7,5 g d'insaponifiable dans les couches éthérées.

- $60 \mathrm{~g}$ d'acides de saponification dans les couches aqueuses (après acidification par $\mathrm{HCl}_{5} \mathrm{~N}$ et extraction à l'éther).

TABLEAU 4

$1 \mathrm{~kg}$ de larves de reines

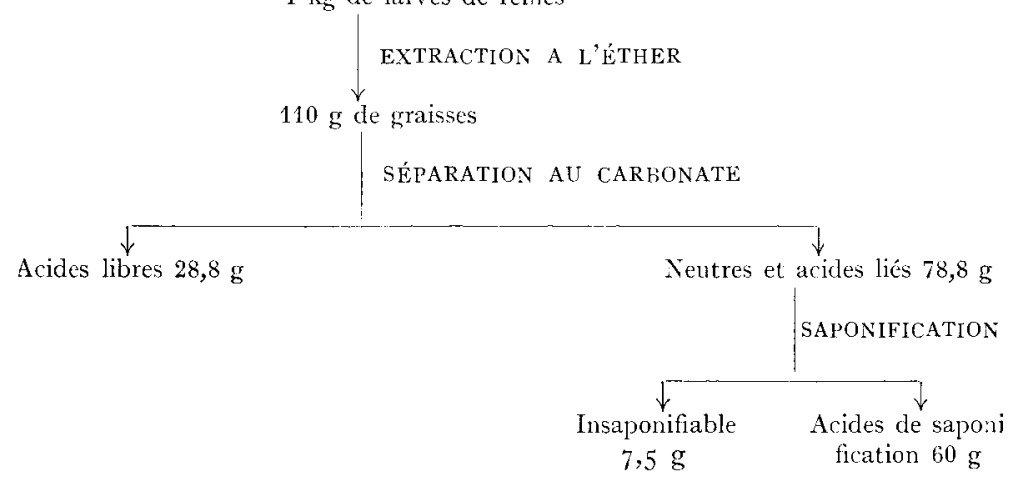

II. ISOLEMENT DES ESTERS DE L'ACIDE HYDROXY-IO DÉCÈNE-2 TRANS OİQUE A PARTIR DES LARVES ROYALES

L'acide hydroxy-Io décène-2 trans oïque a été isolé de la gelée royale par TownSEND et LuCAS (I940) et identifié par BUTENANDT et REMBold (I957). Sa présence dans les glandes mandibulaires des abeilles ouvrières a été démontrée par SIMPSON (rg6o) et par BARbier et PAIN (rg6o, d).

Il était intéressant de rechercher cet acide dans les lipides de larves de reines. On sait en effet que ces larves, dont l'intestin n'est pas ouvert sur l'extérieur, en font une grande consommation. Nous avons ainsi constaté que 1'acide hydroxy-Io décène- 2 trans oïque était présent, mais en faibles quantités, à l'état libre. Ce fait 
nous paraît intéressant, et constitue un élément nouveau pour l'étude du métabolisme de cet acide.

Les acides libres, extraits de leur solution éthérée par le carbonate de sodium $2 \mathrm{~N}$ (voir plan général de fractionnement des larves de reines) ont été chromatographiés sur colonne d'acide silicique Mallinckrodt. Les éluats obtenus peuvent être classés en trois fractions principales, correspondant aux acides palmitique et stéarique, aux esters de 1'acide hydroxy-Iodécène- 2 trans oïque, et enfin aux acides dicarboxyliques.

\section{A) Isolement des acides palmitique et stéarique}

I a première fraction est éluée par le benzène et par le mélange benzène-éther 95,5 . Le spectre IR de cette fraction montre la présence :

- d'un groupe carboxylique (bandes à $3,7,5,8$ et Io, $6 \mu$ ),

- d'un groupe ester (faibles bandes à 5,7 et $8,6 \mu$ ).

Le point de fusion de cette fraction est de $45-48^{\circ}$.

La chromatographie en phase gazeuse à $225^{\circ}$, des esters méthyliques préparés par action d'une solution éthérée de diazométhane, fournit 2 pics dont les volumes de rétention coïncident avec ceux des palmitate et stéarate de méthyle authentiques (fig. I) (1).

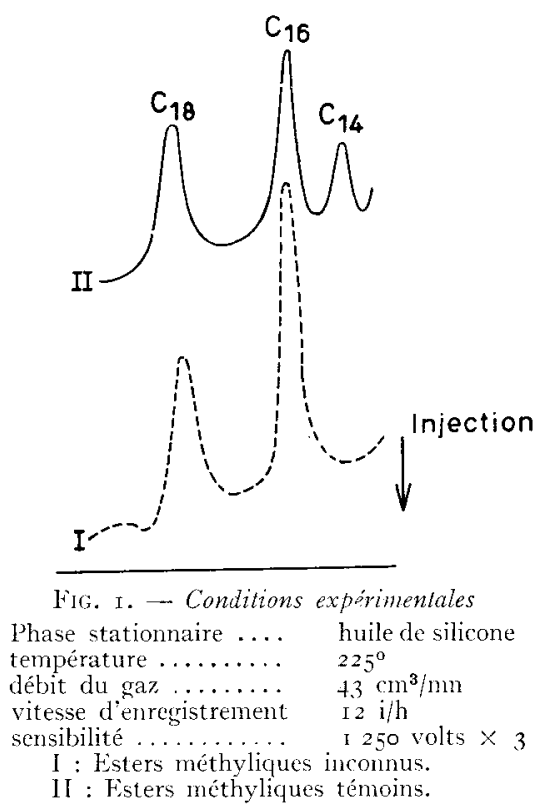

On peut conclure que cette fraction est essentiellement constituée d'acides gras, en mélange avec de petites quantités d'esters (voir ce qui suit).

L'examen des spectres infrarouges et la chromatographie en phase gazeuse des acides obtenus après saponification de ce mélange montrent l'absence de l'acide hydroxy-Io décène-2 trans oïque.

(1) Toutes les mesures de chromatographie en phase gazeuse, ont été effectuées sur un chromatographe PYE, avec comme phase stationnaire l'huile de silicone Dow Corning 5 P. 10o, fixé sur un chromosorb P. 
B) Isolement des esters de l'acide hydroxy-Io décène-2 trans ö̈que

Une seconde fraction est éluée par le mélange benzène-éther 9: I. Nous avons pu montrer que cette fraction était constituée d'esters de l'acide hydroxy-Io décène-2 trans ö̈que avec les acides myristique, palmitique, stéarique et sébacique; 1'acide palmitique étant le principal acide estérifiant 1'acide hydroxylé et l'acide myristique n'étant présent qu'en faibles quantités.

Après cristallisation de cette fraction dans le méthanol, nous avons obtenu un produit dont le point de fusion est de $48-53^{\circ}$.

Son équivalent moléculaire déterminé par titrage est de 560 (calculé par un stéarate d'acide hydroxy-Io décène-2 trans oïque : 452). Cette valeur est calculée par rapport à l'acide stéarique authentique pris comme référence.

Le spectre infrarouge (fig. 2) indique qu'il s'agit d'un acide aliphatique $\alpha \beta$ insaturé, possédant également une fonction ester (bandes à $3,7-5,7$ et $5,8-6,06-8,6$ et Io, $6 \mu$ ).

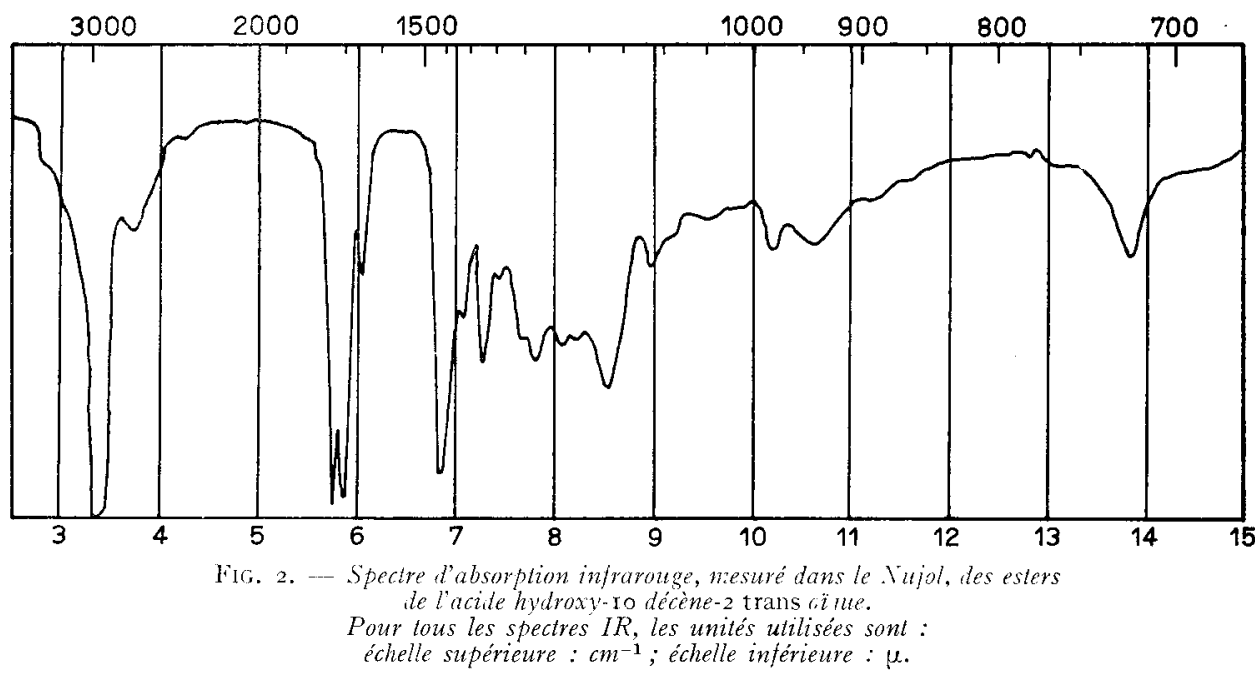

L'analyse élémentaire est en accord avec la formule brute $\mathrm{C}_{26} \quad \mathrm{H}_{48} \mathrm{O}_{4}$ correspondant à un palmitate d'acide hydroxy-ro décène-2 trans oïque.

Le spectre ultraviolet (fig. 3), mesuré dans 1'éther, montre un maximum à $2 \mathrm{I} 2 \mathrm{~m} u$ $(\varepsilon=4900)$ caractéristique d'un acide $\alpha \beta$ insaturé.

Les esters méthyliques, préparés par action d'une solution ethérée de diazométhane, ont été chromatographiés en phase gazeuse. A $200^{\circ}$ aucun pic n'est observable dans les conditions convenant à l'étude des esters d'acides gras de $C_{14}$ à $C_{22}$ (fig. 4).

On constate donc ainsi que le titrage acidimétrique l'avait suggéré, que la substance étudiée possède un équivalent moléculaire assez élevé.

\section{Saponification.}

Nous avons poursuivi l'examen de cette fraction après saponification par la potasse éthanolique à $2,5 \mathrm{p}$. Ioo. Aucun insaponifiable n'est obtenu.

$\mathrm{L}_{1}$ a chromatographie en phase gazeuse de l'ester méthylique de la fraction 


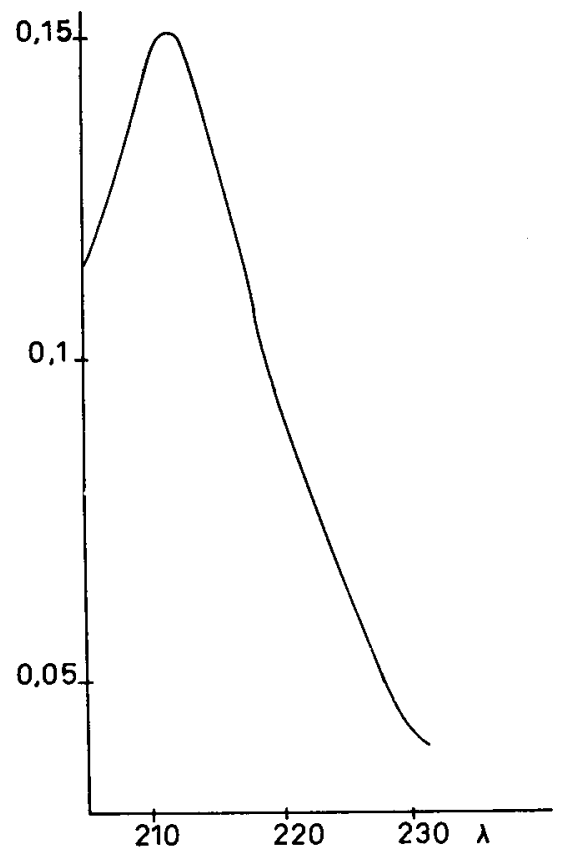

FIG. 3. - Spectre d'absorption ultraviolet des esters de l'acide hydroxy-10 décène-2 trans oüque

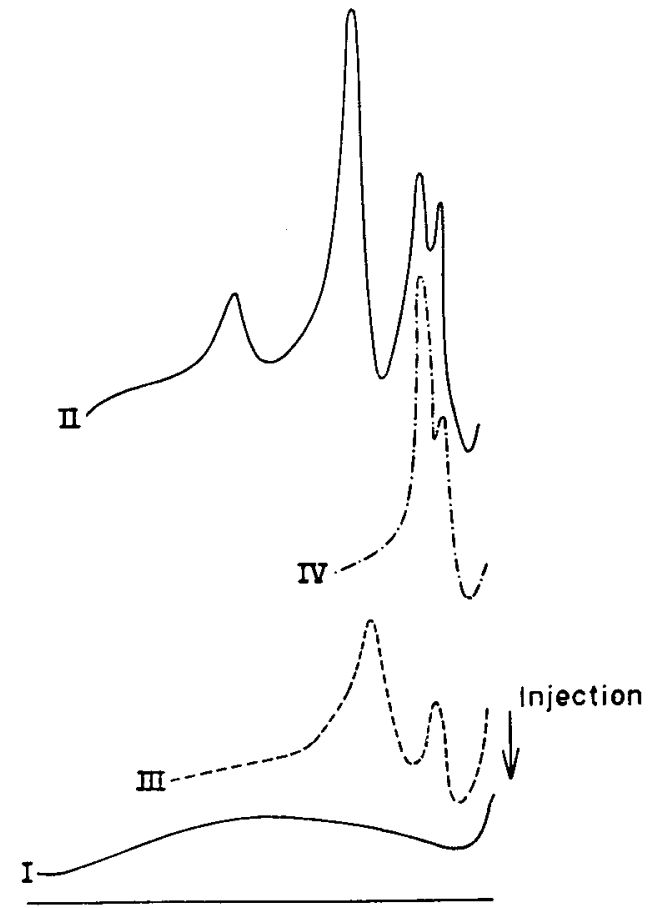

FIG. 4. -... Condilions expérimentales

Phase stationnaire ........huile de silicone

température ...........225

débit du gaz ..........43 $\mathrm{cm}^{3} / \mathrm{mn}$

vitesse d'enregistrement..... $22 \mathrm{i} / \mathrm{h}$

sensibilité ............. I 250 volts $\times 3$

$\mathrm{J}$ : Jister de la fraction avant saponification.

II : Esters de la fraction après saponification.

III : Esters témoins $\mathrm{C}_{14}$ et $\mathrm{C}_{16}$.

IV : Ester témoin de l'acide hydroxy-to décène-z trans oïque contenant un peu d'acide sébacique. 
brute obtenue après saponification, montre qu'il s'agit d'un mélange d'acides gras saturés, $\mathrm{C}_{14} \mathrm{C}_{16}, \mathrm{C}_{18}$, d'acide sébacique, avec de l'acide hydroxy-Io décène-2 trans oïque (fig. 4).

Les acides obtenus après saponification ont été de nouveau chromatographiés sur colonne d'acide silicique, et deux fractions principales ont été obtenues, l'une éluée par le mélange benzène-éther $95: 5$, l'autre par le mélange éther-méthanol $97: 3$.

a) La première fraction est constituée d'un mélange d'acides myristique, palmitique et stéarique. Nous avons déterminé la composition de cette fraction par l'analyse en chromatographie en phase gazeuse.

b) Les éluats obtenus par le mélange éther-méthanol $97: 3$ sont constitués d'acide hydroxy-Io décène-2 trans oïque, contenant une faible quantité d'acide sébacique. Le point de fusion obtenu est de $62-66^{\circ}$, le point de fusion de $1^{\prime}$ acide hydro$\mathrm{xy}$-ro décène- 2 trans oïque authentique étant de $66^{\circ}$.

Le spectre infrarouge (fig. 5) de 1'acide que nous avons isolé, possède les bandes caractéristiques de l'acide hydroxy-Io décène-2 trans oïque (BUTENANot et REMBOI,D (I957) et BARKER, FOSTER et LAMB (I959)).

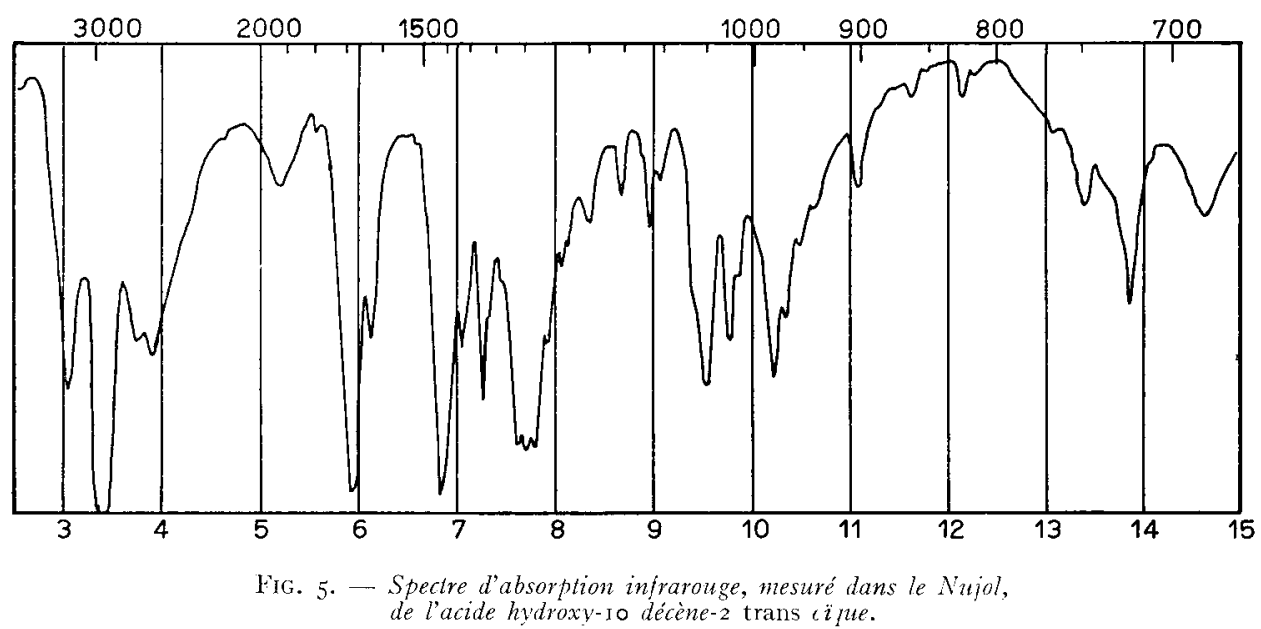

L'équivalent moléculaire, déterminé par titrage est de I96 (calculé par rapport à l'acide palmitique authentique pris comme référence-calculé pour $\mathrm{C}_{10} \mathrm{H}_{18} \mathrm{O}_{3}=\mathrm{I} 86$ ).

L'analyse élémentaire est en accord avec une telle composition.

Le spectre UV dans l'éther montre un maximum à $213 \operatorname{m\mu }(\varepsilon=9$ ooo pour $\mathrm{M}=\mathrm{I} 86$ et $\varepsilon=\mathrm{Io} 400$ pour $\mathrm{M}=\mathrm{I} 96)$.

Nous avons complété ces résultats par 1'analyse chromatographique en phase gazeuse de cette fraction (fig. 6).

Cette fraction contient, comme le témoin, de l'acide hydroxy-Io décène-2 trans oïque et de l'acide sébacique.

Etant donné le faible pic observable en chromatographie gaz liquide, pour le myristate de méthyle, il faut considérer que la fraction étudiée est principalement 
constituée de palmitate, de stéarate et de sébaçate de l'acide hydroxy-Io décène-2 trans oïque.<smiles>[R]C(=O)OCCC=CC(=O)O</smiles>

$\mathbf{R}=$ chaîne de l'acide estérifiant, par exemple :

$\mathrm{CH}_{3}-\left(\mathrm{CH}_{2}\right)_{14}-$ pour un palmitate

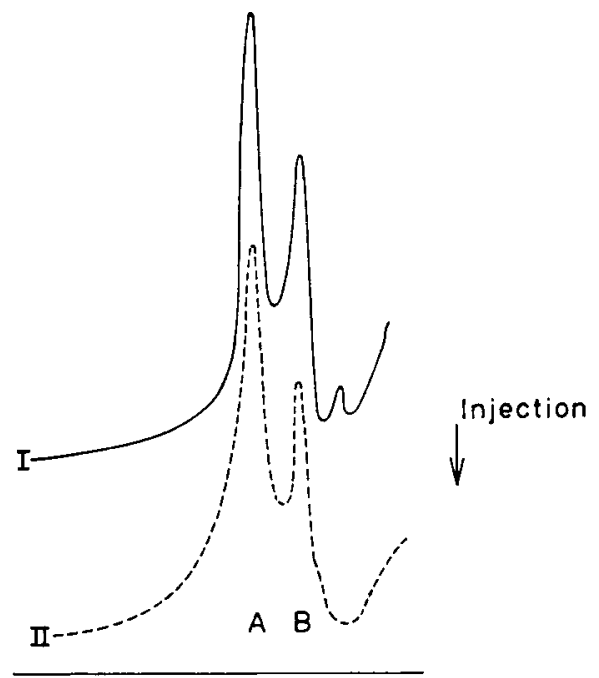

FIG. 6. - Conditions expérimentals.

phase stationnaire ....... huile de silicone

température $\ldots \ldots \ldots \ldots 200^{\circ}$

débit du gaz ...........

vitesse d'cnregistrement.... $18 \mathrm{i} / \mathrm{h}$

sensibilité ............ I 250 volts $x$ 10

I : Esters méthyliques inconnus.

II : Esters méthyliques témoins.

A) Acide hydroxy-ro décène-z trans ö̈que.

B) Acide sébacique.

\section{C) Conclusions}

L'acide hydroxy-Io décène-2 trans oïque de la gelée royale se retrouve dans les larves de reines, mais sous forme estérifiée.

Le rôle biologique de ces esters, pas plus d'ailleurs que celui de l'acide hydroxy-ro décène- 2 trans ö̈que, n'est connu.

La valeur élevée de l'équivalent moléculaire, déterminé par titrage, laisse penser que des esters de poids moléculaires plus élevés que les palmitate ou stéarate sont présents dans cette fraction.

D'autre part, l'existence de polyesters de l'acide hydroxy-Io décène-2 trans oïque (estolides) n'est pas impossible. La formation de tels polyesters a été supposée 
par RoBinson (rg6o), elle expliquerait, selon cet auteur, 1'activité antileucémique de l'acide hydroxy-Io décène-2 trans oïque. L' $\approx$ obtenu pour cette fraction (4 900) est en faveur de cette hypothèse.

\section{D) Partie expérimentale}

Io g de la fraction "acides libres" (chzpitre I) ont été chrom ztographiés sur colonne de $350 \mathrm{~g}$ d'acide silicique Mallinskrodt. Les élutions ont été effectuées par fractions de $200 \mathrm{ml}$.

\begin{tabular}{|c|c|}
\hline I à ro benzène & $5970 \mathrm{mg}$ \\
\hline I I à I4 benzène-éther $95: 5 \ldots$ & $1927 \mathrm{mg}$ \\
\hline I 5 à r 8 benzène-éther $9: 1 \ldots$ & $\mathrm{I} \mathrm{I}^{6} 6 \mathrm{mg}$ \\
\hline I9 à 2 I benzène-éther $8: 2 \ldots$ & I64 \\
\hline 22 à 24 benzène-éther I : I $\ldots \ldots \ldots$ & I08 $\mathrm{mg}$ \\
\hline 25 à 27 éther & $462 \mathrm{lng}$ \\
\hline 28 à 30 éther & 80 \\
\hline
\end{tabular}

Pour les examens en phase gazeuse, I mg de substance a été repris par une petite quantité (environ $0,5 \mathrm{ml}$ ) d'une solution éthérée de diazométhane. Après un contact de quelques minutes, le produit est amené à sec.

\section{a) Étude des fractions I $\dot{a}$ Io}

Les spectres IR de ces fractions, mesurés dans le Nujol, indiquent qu'il s'agit d'acides aliphatiques, contenant une faible proportion d'esters. Par cristallisation dans le méthanol, on obtient des cristaux : $\mathrm{F}=45^{-4} 8^{\circ}$.

La chromatographie en phase gazeuse des esters de ces fractions montre la présence des esters palmitique et stéarique (fig. I).

\section{Saponification.}

La saponification de $3 \mathrm{~g}$ de cette fraction a été réalisée. On dissout le produit dans $20 \mathrm{ml}$ d'éthano] à chaud. Après addition de $30 \mathrm{ml}$ de potasse éthanolique à $5 \mathrm{p}$. 100 , la solution a été portée 2 heures au reflux. Après refroidissement, on extrait à l'éther et on acidifie ensuite la couche aqueuse par une solution d'acide chlorhydrique $2 \mathrm{~N}$. On ajoute $50 \mathrm{ml}$ d'eaul, et on extrait 3 fois par $40 \mathrm{ml}$ d'éther.

Ces derniers extraits éthérés sont lavés 3 fois par $20 \mathrm{ml}$ d'eaul, séchés sur sulfate de soude et amenés à sec. On obtient ainsi $2,9 \mathrm{~g}$ d'acides

Ces $2,9 \mathrm{~g}$ d'acides ont été chromatographiés sur $100 \mathrm{~g}$ d'acide silicique. Les élutions ont été réallisées par fractions de $5 \circ \mathrm{ml}$.

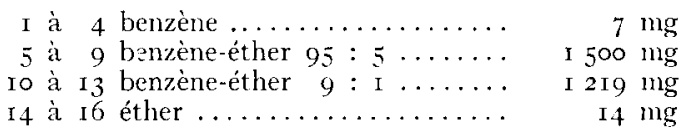

L'examen par chromatographie en phase gazeuse des esters méthyliques de ces fractions, ne montre pas la présence de nouveaux acides et en particulier d'hydroxy-acides.

\section{b) Étule des fractions $\mathrm{I}_{5} \dot{a} \mathbf{1} 8$}

Nous avons cristallisé 2 fois cette fraction, la première fois dans le méthanol, la seconde fois dans le mélange éther-pentane. Le produit fond à $48-53^{\circ}$.

Le spectre IR (fig. 2), mesuré dans le Nujol, indirque lis présence des bandes caractéristiques des fonctions acide et ester aliphatiques.

Nous avons effectué sur ce composé un titrage d'acidité qui a donné les résultats suivants :

I $\mathrm{mg}$ de ce composé consomme $96 \mathrm{~mm}^{3}$ de potassé éthanolique $\mathrm{N} / 5^{\circ}$;

$2,3 \mathrm{mg}$ de ce composé consomment $225 \mathrm{~mm}^{3}$ de potasse éthanolique $\mathrm{N} / 50$;

$2,3 \mathrm{mg}$ d'acide stéarique témoin consomment $445 \mathrm{~mm}^{3}$ de potasse éthanolique $\mathrm{N} / 50 \mathrm{P} \mathrm{MI}=284$.

Nous obtenons la valeur de 560 .

L'injection des esters méthyliques dans le chromatographe gaz. liquide ne fournit aucun pic jusqu'à la position correspondant à l'ester $\mathrm{C}_{22}$ (fig. 4).

Annales de l'Abeille. $-19^{6} 3$. 
L'analyse élémentaire est en accord avec la formule brute : $\quad \mathrm{C}_{26} \mathrm{H}_{48} \mathrm{O}_{4}$.

$$
\begin{array}{ccccc}
\text { trouvé } & \mathrm{C} \% & 73,20 & \mathrm{H} \% & \mathrm{II}, \mathrm{I} 2 \\
\text { calculé } & & 73,5,3 & & \mathrm{II}, 39
\end{array}
$$

Le spectre U V mesuré dans l'éther ( $\mathrm{r}$ mg dans $60 \mathrm{ml}$ d'éther) possède un maximum à 2 I $2 \mathrm{~m} \mu$ (fig. 3).

On a donc $\varepsilon=\frac{0,146 \times 560}{0,00166 \times 10}=4900$

Quelques tests ont été faits sur ce produit. Le test à l'hypoiodite de sodium et l'essai de précipitation d'une 2-4-dinitrophénylhydrazone ont été tous deux négatifs.

\section{Saponification.}

$806 \mathrm{mg}$ de cette fraction, dissous dans $20 \mathrm{ml}$ d'éthanol, ont été saponifiés par chauffage au reflux pendant i heure, en présence de $\mathrm{KOH}$ à $5 \mathrm{p}$. Ioo; après extraction par l'éther, la fraction aqueuse est acidifiée par $\mathrm{HCl} 2 \mathrm{~N}$. On l'extrait ensuite 2 fois à l'éther et on obtient $737 \mathrm{mg}$ d'extrait. $30 \mathrm{ml}$.

Nous avons chromatographié ces acides sur $50 \mathrm{~g}$ d'acide silicique, en éluant par fractions de

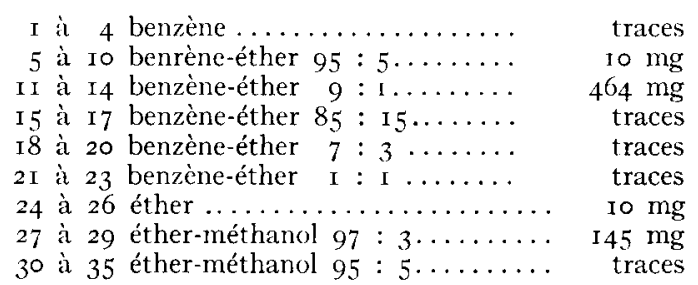

Le passage des esters méthyliques de la fraction i I à i 4, éluée par le mélange benzène-éther 95: 5, dans la chromatographe gaz-liquide, permet d'identifier un mélange d'esters d'acides $\mathrm{C}_{14}$, $C_{16}, C_{18}$ et sébacique, dans lequel l'ester palmitique est le principal composé. Le spectre IR des fractions de 27 à 29 éluées par le mélange éther-méthanol $97: 3$ (après cristallisation de ces fractions dans le mélange éther-pentane) est convenable pour la structure de l'acide hydroxy-1o décène-2 trans oïque (fig. 5).

Nous avons mesuré le spectre UV ( $r, 8 \mathrm{mg}$ dans $100 \mathrm{ml}$ d'éther ).

$$
\varepsilon=\frac{0,955}{0,0018 \times 10^{-}}=10400
$$

Nous avons effectué sur ce composé un titrage d'acidité qui a donné une valeur de 196 pour l'équivalent moléculaire.

I, I mg consomment $90 \mathrm{~mm}^{3}$ de potasse éthanolique $\mathrm{N} / 5^{\circ}$

I,6 mg d'acide palmitique témoin consomment $100 \mathrm{~mm}^{3}$ de potasse éthanolique $\mathrm{N} / 50 \mathrm{PM}=256$;

L'analyse élémentaire $\mathrm{C}_{10} \mathrm{H}_{18} \mathrm{O}_{3}$ donne :

$$
\begin{array}{lllll}
\text { Calculé } & \mathrm{C} \% & 64,49 \\
\text { Trouvé }
\end{array} \quad \mathrm{IL} \% \quad \begin{array}{r}
9,74 \\
64,20
\end{array} \quad \begin{array}{r}
9,92 \\
\end{array}
$$

L'injection dans le chromatographe gaz-liquide, des esters méthyliques de cette fraction, met en évidence un pic principal, dont le volume de rétention est identique à celui de l'ester de l'acide hydroxy-Io décène- 2 trans oïque authentique; on observe un second pic correspondant à une certaine quantité d'acide sébacique (fig. 6).

\section{III. - ÉTUDE DES ACIDES DICARBOXYLIQUES}

Deux acides dicarboxyliques : 1'acide sébacique et 1'acide décène-2 trans dioïque, ont précédemment été isolés de la gelée royale (voir introduction). Nous avons pensé que ces acides, et peut-être d'autres, devaient se retrouver dans les larves de reines. Ces acides présentent un intérêt du point de vue biologique ; en effet les travaux de 
TownSEND (I960) ont montré sur la Souris, l'activité antileucémique de ces acides. Il est donc possible qu'ils participent à l'activité antileucémique totale de la gelée royale.

Une hypothèse récente de Sir Robert RoBinson (r960) suppose que l'activité antileucémique de la gelée royale serait en rapport avec la possibilité des composés présents à former des estolides (il s'agirait donc d'un effet physique). Le fait que nous ayons isolé des esters de l'acide hydroxy-ro décène-2 trans oïque, à poids moléculaire relativement élevé, serait en accord avec cette hypothèse.

Nous avons donc recherché ces acides dicarboxyliques, dans les larves de reines d'une part, et dans la gelée royale d'autres part.

\section{A) Dans les larves royales}

Les acides dicarboxyliques étant plus fortement retenus sur acide silicique, nous les avons principalement recherchés en fin de chromatographie sur colonne.

Nous avons effectué la recherche de ces acides, sur les acides libres d'une part, et sur les acides obtenus après saponification d'autre part. Nous ne les avons trouvés que dans les acides libres.

Dans la chromatographie des acides libres (voir II) nous avons obtenu une importante fraction, éluée par l'éther $(462 \mathrm{mg})$. Nous avons essayé de cristalliser cette fraction, en la dissolvant dans l'éther, et en ajoutant du pentane à $0^{\circ}$.

\section{a) Isolement de l'acide décène-2 trans diö̈que.}

Nous avons d'abord isolé $5 \mathrm{mg}$ d'une fraction insoluble dans l'éther. Le point de fusion F : I60-I $70^{\circ}$ et le spectre UV (maximum à $2 \mathrm{I} 5 \mathrm{~m} \mu$ ) nous ont fait penser à l'acide décène-2 trans dioïque isolé précédemment par Brown et FrEURE (I959).

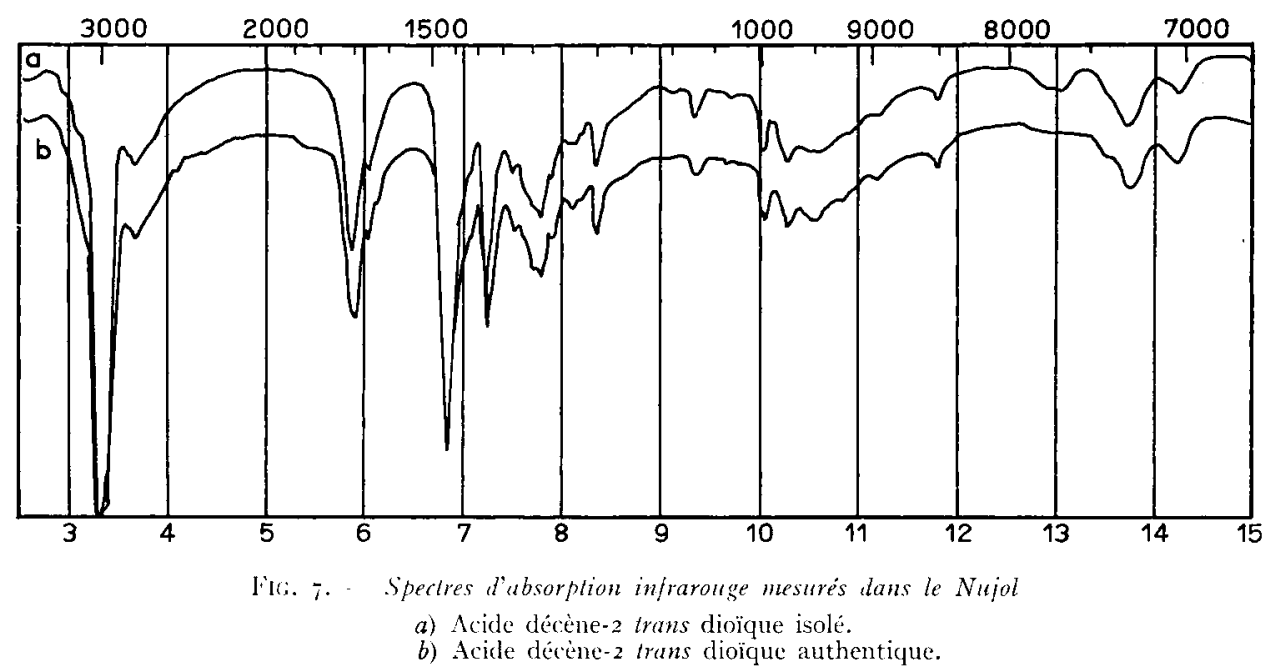

En fait, le point de fusion de mélange avec un acide décène-2 trans dioïque authentique ne présente pas de dépression $\mathrm{F}$ : $\mathrm{x} 60-\mathrm{I} 70^{\circ}$; de plus la comparaison des spectres infrarouges (fig. 7) confirme cette identité. 
b) Identification des acides subérique et sébacique.

Le reste de la fraction a pu être cristallisé dans le mélange éther-pentane, e! nous avons obtenu $225 \mathrm{mg}$ de cristaux. Le spectre infra-touge des produits cristallisés montre qu'il s'agit d'un mélange d'acides contenant encore de l'acide décène-2 trans dioïque.

Nous avons alors étudié ces cristaux par chromatographie sur papier en utilisant la technique décrite par BROWN, FELAUER et FREURE (I96I).

Nous avons obtenu les $\mathrm{R}_{f}$ suivants :

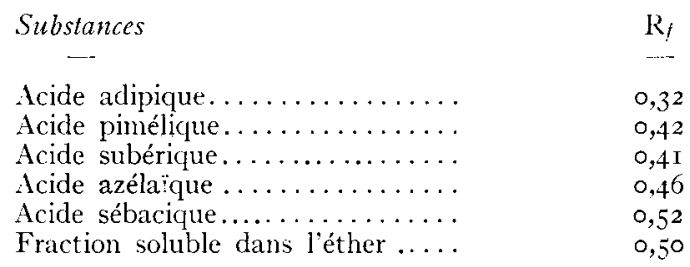

Cette séparation sur papier étant mauvaise, nous avons alors étudié ces fractions par chromatographie en phase gazeuse. A partir d'esters préparés par action d'une solution éthérée de diazométhane, deux séries de chromatographies ont été effectuées l'une à $200^{\circ}$ et l'autre à $150^{\circ}$.

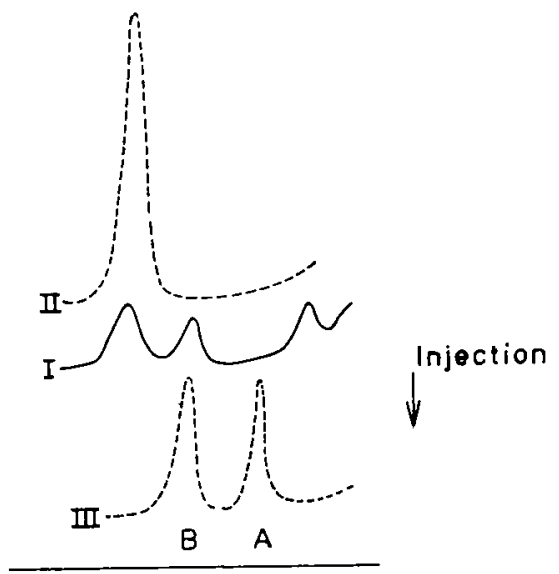

\footnotetext{
l'Ic. S. - Conditions expérimentales

phase stationnaire ....... huile de silicone

température ..........200"

débit du gaz $\ldots \ldots \ldots \ldots .26 \mathrm{~cm}^{3} / \mathrm{mm}$

vitesse d'enregist rement.... Is $\mathrm{i} / \mathrm{h}$

semsibilite ........... I 250 volts $\% 10$

II : Acide décène-2 trans dioïque authentique.

111 : Acides azélaique $(S)$ et séhacique (B) authentiques.
}

I : lraction à cetudier.

Dans la chromatographie à $200^{\circ}$ nous avons observé la présence de trois pics. Les volumes de rétention de deux d'entre eux sont identiques aux volumes de rétention des sébaçate et décène- 2 trans dioate de méthyle authentiques (fig. 8). 
L'acide hydroxy-Io décène-2 trans oïque, ayant un volume de rétention peu différent de celui de l'acide décène- 2 trans diö̈que, nous avons vérifié l'absence de cet acide, dans cette partie de chromatographie, par répétition des essais en présence de témoins internes (fig. 9).

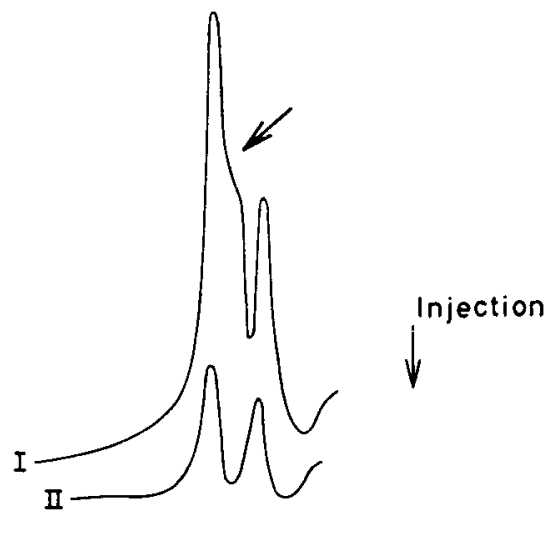

FIG. 9. - Conditions expérimentales

phase stationnaire ......... huile de silicone

température ............200

débit du gaz $\ldots \ldots \ldots \ldots \ldots .32 \mathrm{~cm}^{3} / \mathrm{mm}$

vitesse d'enregistrement...... I $8 \mathrm{i} / \mathrm{h}$

sensibilités.............. I 250 volts $\times 3$

I : Fractions à étudier, contenant en phus de l'acide hydroxy-ro décène-2 trans oïque.

II : Fraction à étudier, contenant en plus de l'acide décene-2 trans dioique.

Le volume de rétention du troisième pic est identique, à $I 5^{\circ}$, à celui du subérate de méthyle (fig. Io).
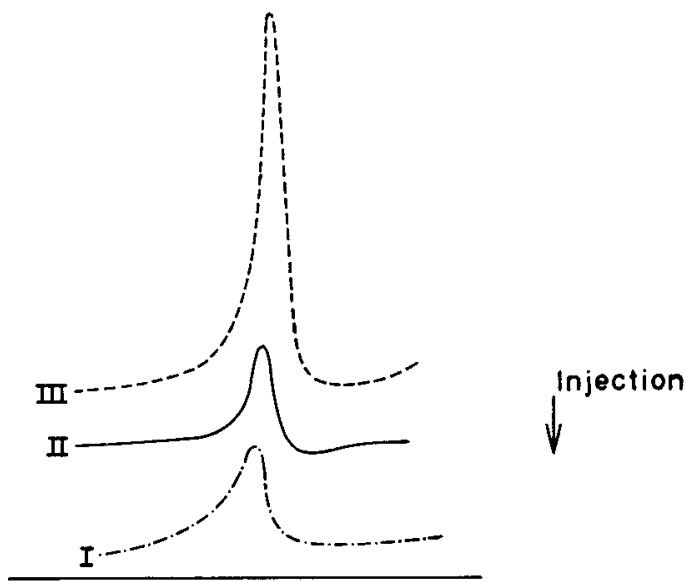

I : Subérate de méthyle.

II : Fraction à étudier.

III : Fraction à étudier contenant en plus du subérate de méthyle. 
Il est probable que les acides dicarboxyliques mis en évidence dans la gelée royale (voir ci-après) sont également présents dans les larves royales. Mais par suite d'un phénomène de dilution, dû à la présence de nombreuses autres substances lipidiques, il n'a pas été possible de les identifier.

\section{B) Dans la gelée royale}

L'extraction chloroformique de $8 \mathrm{~g}$ de gelée royale, séchée 24 heures sous vide à $20^{\circ}$, fournit environ $500 \mathrm{mg}$ d'extrait. Une séparation par le carbonate de sodium fournit ensuite $187 \mathrm{mg}$ de fraction acide, que nous avons chromatographié sur acide silicique.

Les points de fusion de ces fractions sont de $60-80^{\circ}$.

Nous avons ensuite, dans les mêmes conditions que pour les larves royales, examiné ces fractions en chromatographie en phase gazeuse. Seules, les fractions éluées par les mélanges benzène-éther $I:$ I et $3: 7$ contiennent des acides dicarboxyliques.

Nous avons identifié les acides suivants :

a) Dans les fractions éluées au mélange benzène-éther I : I (fig. II) les acides

$$
\begin{aligned}
& \text { pimélique } \\
& \text { subérique } \\
& \text { sébacique. }
\end{aligned}
$$

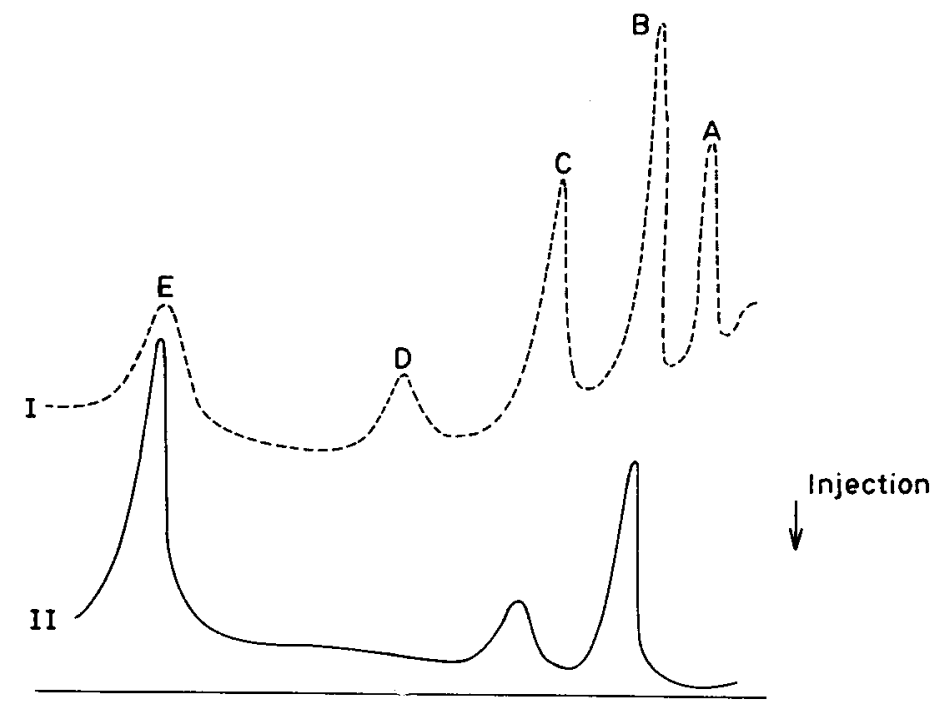

FIG. II. - Conditions expérimentales

phase stationnaire .........huile de silicone

température ............ I $50^{\circ}$

débit du gaz .......... I $\mathrm{cm}^{3} / \mathrm{mm}$

vitesse d'enregistrement....... $12 \mathrm{i} / \mathrm{h}$

sensibilité .............. I 500 volts $\times$ Io

I : Témoins : adipate $(A)$ - pimélate $(B)$ - subérate $(C)$ - azélaate (D) -- sébaçate (E).

II : Fraction éluce au mélange benzène-éther $\mathrm{I}: \mathrm{I}$. 
b) Dans les fractions éluées par le mélange benzène-éther $3: 7$ nous avons isolé les acides (fig. I2).
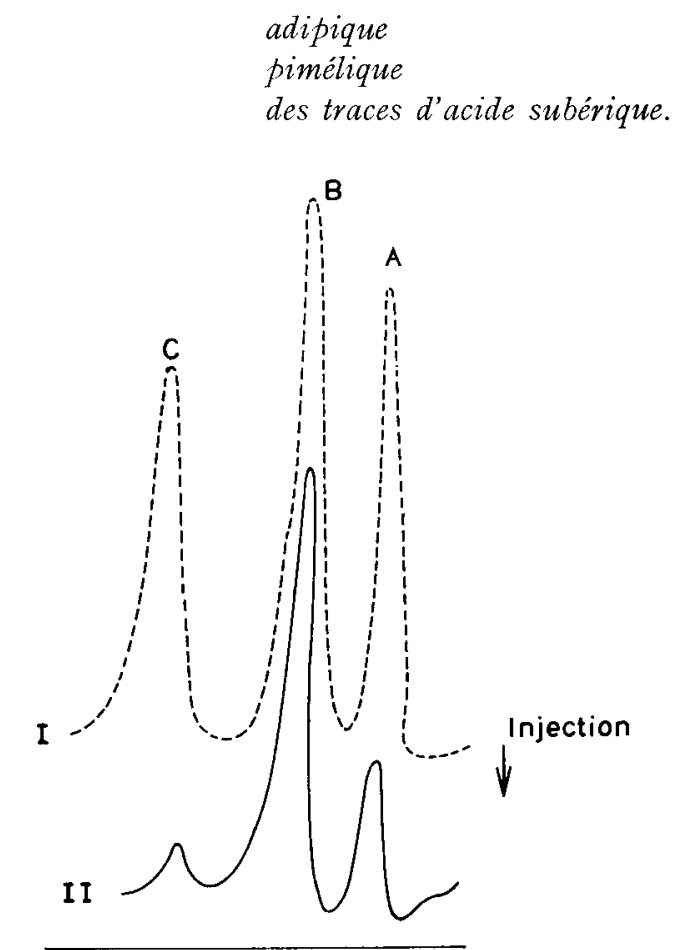

FIG. I2. - Conditions expérimentales phase stationnaire ......... huile de silicone température ........... I $50^{\circ}$ débit du gaz ............. $\mathrm{cm}^{3} / \mathrm{mn}$ vitesse d'enregistrement...... $2 \mathrm{i} / \mathrm{h}$ sensibilité .............. I 250 volts $\times$ ro

I : Témoins : adipate $(A) \cdots$ pinélate $(B)$--. subérate $(C)$.

II : Fraction éluée au mélange benzène-éther $3: 7$.

L’identification de ces substances a été confirmée par la répétition des chromatographies, après introduction de témoins internes dans les échantillons.

Nous avons donc identifié, dans la gelée royale, les acides : adipique, pimélique, subérique, sébacique.

Seuls les acides sébacique et décène-2 trans diö̈que étaient précédemment cités dans la littérature.

L'évaluation des quantités présentes n'est pas aisée ; cependant la comparaison des pics obtenus, avec celui de l'ester sébacique, permet de déduire les relations suivantes :

Les nouveaux acides identifiés sont en quantités inférieures à celle de l'acide sébacique. (La littérature donne pour cet acide $0,02 \mathrm{p}$. Ioo par rapport au poids sec de la gelée royale.) Les acides pimélique et subérique sont en quantités plus importantes que 1'acide adipique, qui n'existe qu'en très faibles proportions. Cependant si on compare la somme des trois acides adipique, pimélique et subérique, on constate que cette somme est au moins égale à la quantité d'acide sébacique. 


\section{C) Conclusions}

Les larves de reines, et la gelée royale, contiennent donc une série d'acides dicarboxyliques. Parmi ces acides, les acides adipique, pimélique et subérique n'avaient jamais été signalés.

Une activité antileucémique sur la Souris ayant été observée par TownsEnD (Ig60) pour ces acides, la présence de cette série d'acides est un fait biologiquement intéressant.

\section{D) Partie expérimentale}

\section{a) Etude des acides dicarboxyliques des larves royales}

Dans la chromatographie des acides libres (chapitres I et II), nous avons obtenu en fin de chromatographie 4 fractions éluées par l'éther, soit $462 \mathrm{mg}$.

\begin{tabular}{|c|c|c|c|c|}
\hline fractions & éluant & $\mathrm{mg}$ & $\begin{array}{l}\text { cristallisation } \\
\text { éther-pentane }\end{array}$ & $\mathrm{PF}$ \\
\hline$\ldots$ & - & - & - & - \\
\hline 25 & éther & 98,7 & 25,8 & I $27-\mathrm{I} 48^{\circ}$ \\
\hline 26 & éther & $16 \mathrm{I}, 7$ & $\mathrm{I} 2 \mathrm{I}$ & $13^{2-1} 4^{\circ}$ \\
\hline 27 & éther & 108,3 & $5^{6,8}$ & $13^{\circ-1} 5^{\circ}$ \\
\hline 28 & éther-méthanol & 98,3 & 22 & $130-15^{0}$ \\
\hline
\end{tabular}

I) Les fractions 26 et 27 ne sont pas entièrement solubles dans l'éther, et on peut isoler à l'état cristallisé $5 \mathrm{mg}$ de fraction insoluble.

Le spectre infrarouge de ces $5 \mathrm{mg}$ est identique à celui de l'acide décène-2 trans dioïque (fig. 7) et le spectre ultraviolet, mesuré dans le chloroforme ( $\mathrm{mg}$ de produit dissous dans $60 \mathrm{ml}$ ) présente un maximum à $215 \mathrm{~m} \mu$.

Le point de fusion de cet acide, $160 \cdot 170^{\circ}$, est identique au point de fusion de l'acide décène-2 trans dioïque authentique, et le point de fusion de mélange ne présente pas de dépression.

2) Les cristaux obtenus par cristallisation dans le mélange éther-pentane ont été étudiés :

- Par chromatographie sur papier.

Le solvant de migration utilisé est un mélange :

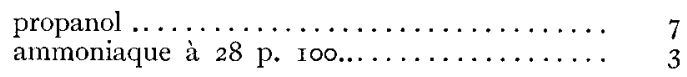

La durée de migration est d'environ 36 heures.

La révélation se fait à l'aide d'une solution de vert de bromocrésol.

- Par chromatographie en phase gazeuse des esters méthyliques préparés à l'aide d'une solu. tion de diazométhane.

Nous avons effectué ces analyses :

à $150^{\circ}$ pour l'acide subérique (fig. Io);

à $200^{\circ}$ pour les acides sébacique et décène- 2 trans-dioïque (fig. 8 et 9).

\section{b) Étude des acides dicarboxyliques de la gelée royale}

$8 \mathrm{~g}$ de gelée royale ont été extraits au chloroforme par ébullition à reflux pendant I 2 heures. On sépare les deux couches, on sèche sur sulfate de soude la couche chloroformique, on évapore le chloroforme et on obtient environ $500 \mathrm{mg}$ de résidu.

L'extrait ainsi obtenu est ensuite dissous dans $60 \mathrm{ml}$ de mélange chloroforme-éther I : 3 .

On sépare les acides par extraction de cette solution par 2 fois $30 \mathrm{ml}$ d'une solution de carbonate de sodium $2 \mathrm{~N}$. On lave une fois par $30 \mathrm{ml}$ d'eau. Après acidification des hydrosolubles réunis, par une solution d'acide chlorhydrique $2 \mathrm{~N}$, on extrait 3 fois par $50 \mathrm{ml}$ du mélange chloroformeéther I : 3 . La couche organique a été lavée une fois par $30 \mathrm{ml}$ d'eau, et séchée sur sulfate de sodium.

Nous avons obtenu $224 \mathrm{mg}$. 
Chromatographie sur colonne.

Ces $224 \mathrm{mg}$ ont été chromatographiés sur une colonne de $\mathrm{I}$ o g d'acide silicique Mallinckrodt. Les éluats sont de $25 \mathrm{ml}$.

\begin{tabular}{clc} 
fractions & \multicolumn{1}{c}{ éluant } & $\mathrm{mg}$ \\
I & benzène & Io $\mathrm{mg}$ \\
4 à 6 & benzène-éther $\mathrm{I}: \mathrm{I}$ & $143 \mathrm{mg}$ \\
7 à 9 & benzène-éther $3: 7$ & $3 \mathrm{mg}$ \\
10:1 12 & éther & $0 \mathrm{mg}$ \\
I3 $\mathrm{à} 5$ & éther-méthanol $9: 1$ & traces
\end{tabular}

L'étude par chromatographie en phase gazeuse a été effectuée sur les fractions I à 3,4 à 6 et 7 à 9. Jes résultats ont été obtenus avec ces deux dernières fractions.

\section{IV. - RECHERCHE, DU p-HYDROXY BENZOATE DE MÉTHYLE}

BROWN, FELAUER et FREURE (I96r) ont décrit l'isolement de l'acide $p$-hydroxy benzoïque à partir de la gelée royale. Cet isolement ayant été effectué après saponification, il était possible de penser à la présence du p-hydroxy benzoate de méthyle (Nipagine) précédemment isolé des extraits totaux de reines d'abeilles par BARBIER, LEDERER, REICHSTEIN et SCHINDLER (I960).

Nous avons utilisé deux méthodes différentes pour rechercher le p-hydroxy benzoate de méthyle des extraits de gelée royale d'une part, et des larves de reines, d'autre part :

a) La chromatographie sur papier, dans le système chloroforme-formamide selon la technique de ZaFraroni, Burtor et Keutmann (I950).

b) La chromatographie en phase gazeuse à $\mathrm{I} 60$ et $200^{\circ}$. Ces deux méthodes ont permis de constater l'absence du p-hydroxy benzoate de méthyle, dans les deux extraits examinés.

\section{Conclusion}

L'acide $p$-hydroxy benzoïque, isolé pat BRown, FELAUER et FREURE (I96I), existe donc comme tel dans la gelée royale.

\section{Partie expérimentale}

Pour la chromatographie sur papier par la méthode ascendante, du $p$-hydroxy benzoate de méthyle, nous avons utilisé un papier Whatman $n^{0} \mathrm{I}$.

Cette feuille a été trempée dans le mélande formamide-acétone $\mathrm{I}: 3$. Jille a été ensuite séchée entre deux feuilles de papier-filtre.

Nous avons utilisé comme solvant de développement le sytème chloroforme-formamide $7: 3$.

Après séchage du chromatogramme, les taches correspondant au p-hydroxy-benzoate sont visibles en lumière ultraviolette (taches sombres). le $R_{f}$ est voisin de 0,5 .

\section{V. - PROBlìme DES STÉROLS ALIMENTAIRES DES LARVES ROYALES}

Clark et Bloch (I959 $a, b, c$ ont étudié la biogénèse des stérols chez les insectes. Ils ont montré que les insectes ne peuvent vivfe en absence de stérols, qu'ils ne peuvent par ailleurs synthétiser.

Or, ainsi que l'a montré von RHEIN (I933), les larves de reines d'abeilles sont nourries uniquement de gelée royale, et la présence de stérols ne paraît pas y_avoir 
été signalée. Les larves d'ouvrières reçoivent, les deux premiers jours de leur existence, de la gelée royale, puis ensuite du pollen mélangé à la gelée royale. On pouvait se demander quelle pouvait être l'origine des stérols chez les larves de reines, à moins d'envisager un développement en absence de stérols.

Nous avons tenu à vérifier le fait connu que les larves royales ne reçoivent pas de pollen. Nous remercions M. Chauvin (Station de Recherches sur l'Abeille et les Insectes sociaux de Bures-sur-Yvette) d'avoir bien voulu rechercher le pollen dans la gelée royale d'une part et dans les intestins de larves d'autres part.

Ces recherches ayant été négatives, nous avons alors cherché à mettre en évidence une fraction stérolique dans les insaponifiables de larves royales. Ayant, par chromatographie sur colonne d'acide silicique, isolé une telle fraction, nous en avons étudié la composition. La présence du méthylène-24 cholestérol apporte une solution au problème posé.

\section{A) Isolement du méthylène-24 cholestérol à partir des larves royales}

$600 \mathrm{mg}$ de la fraction insaponifiable ont été chromatographiés sur acide silicique Mallinckrodt.

L'examen systématique de tous les éluats obtenus, par la réaction de Liebermann et par chromatoplaques, a montré la présence d'une importante fraction stérolique, $200 \mathrm{mg}$, éluée par le mélange benzène-éther $95: 5$.

Après deux cristallisations dans le méthanol, nous avons obtenu $45 \mathrm{mg}$ de plaques incolores $\mathrm{F}$. I 40-I $43^{\circ}$. La réaction de Liebermann donne une coloration vert très foncée, elle est donc très positive.

Ce stérol a été identifié au méthylène-24 cholestérol le stérol principal des abeilles (Barbier et Schindier i959) et du pollen (Barbier, Huger, et Lederer ig6o).

Le pouvoir rotatoire, mesuré dans le chloroforme, est de :

$$
(x)_{1}^{20}=-38 \pm 2^{0} \quad(c=0,8)
$$

Le point de fusion de mélange avec un méthylène-24 cholestérol authentique ne présente pas de dépression F. I $4 \mathrm{I}-\mathrm{I} 44^{\circ}$.

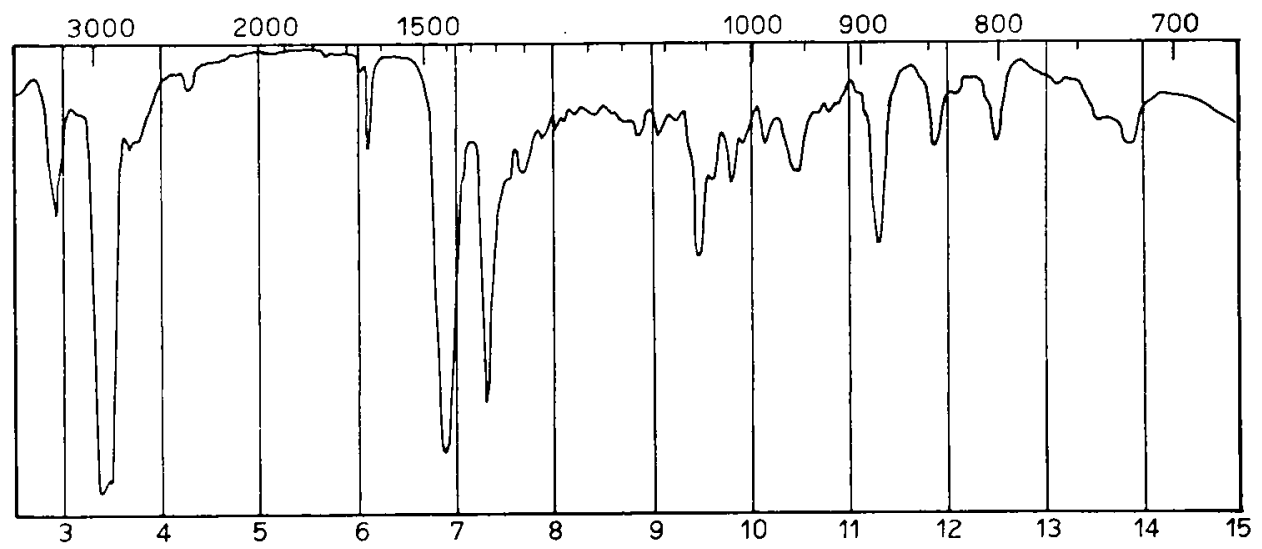

FIG. I3. - Spectre d'absorfion infrarouge, mesuré dins le Nujol, du méthylène-24 cholestérol 
Le spectre IR (fig. I3) présente bien les bandes caractéristiques du groupe méthylènique à 6 ,I et $I I, 3 \mu$; et il est identique au spectre IR du méthylène 24 cholestérol publié par BARBIER et SCHINDLER (I959). Le $\mathrm{R}_{f}$ sur chromatoplaques (système pentane-acétate d'éthyle $85:$ I5) est identique à celui d'un méthylène-24 cholestérol authentique $\left(\mathrm{R}_{f}=0,23\right)$ (fig. 15$)$.

Nous avons de plus préparé deux dérivés :

a) Un acétate. - Par action de l'anhydride acétique dans la pyridine.

$$
\text { F.I3I-I } 35^{\circ} \quad(\alpha)_{\mathrm{D}}^{20}=-46 \pm 2^{\mathrm{o}} \quad(\mathrm{c}=0,8 \text { chloroforme })
$$

Le point de fusion de mélange avec un acétate authentique ne présente pas de dépression F. I3I-I $35^{\circ}$ (le point de fusion de cet acétate étant de $\mathrm{I} 3 \mathrm{I}-\mathrm{I} 36^{\circ}$ ).

Le spectre IR présente bien les bandes à 6, I et II, $3 \mu$ ainsi que les bandes à 5,8 et $8, \mathbf{I} \mu$ (groupe acétate).

b) Un acétate de céto-24 cholestérol. - Nous avons obtenu ce produit par ozonisation de l'acétate de méthylène-24 cholestérol.

Le spectre IR (fig. I4) montre la disparition des bandes caractéristiques du groupe méthylénique $(6$, I et II,3 $\mu)$ et l'apparition d'une bande nouvelle due au groupement cétonique en $24(5,9 u)$. Le point de fusion I22-I $26^{\circ}$ est en accord avec la valeur signalée dans la littérature F. I27 (IDLER et FAGERI,UXD I955).

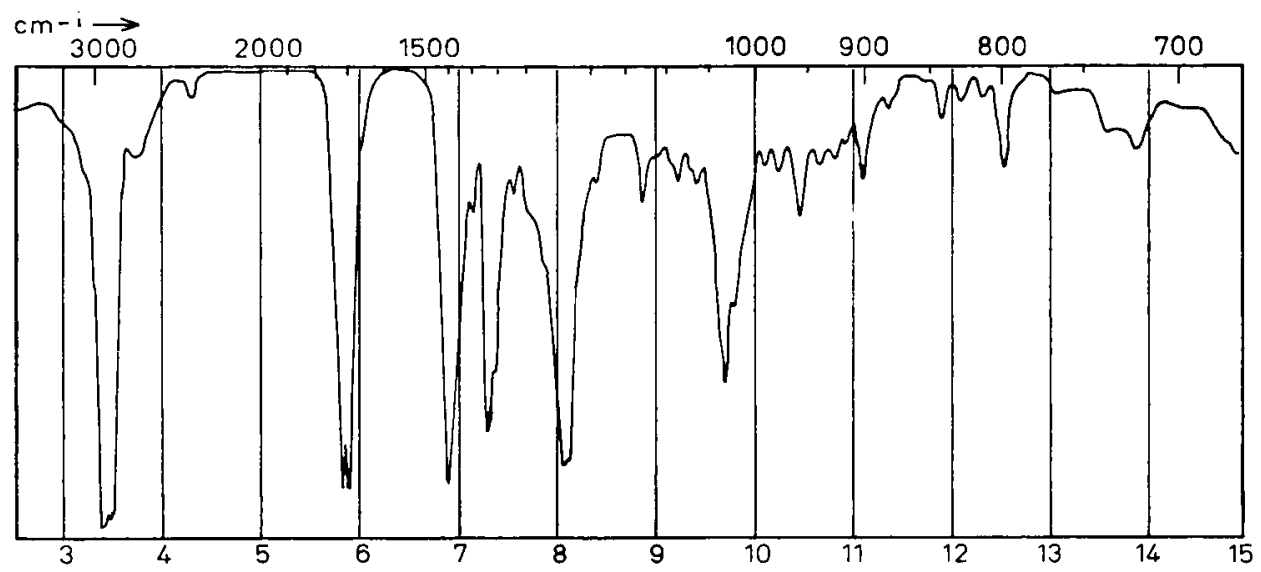

FIG. 34. - Spectre d'absorption intrarouge, mesuré dans le liujol, de lacétute de céto-2+ cholestérol

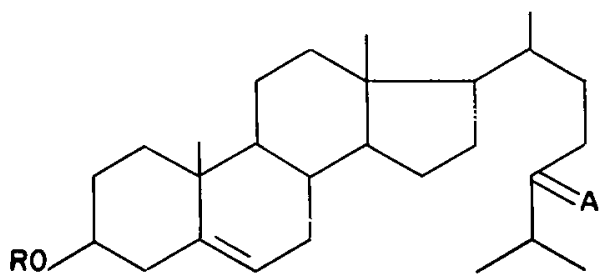
$\frac{\mathrm{R}}{=}=\mathrm{H}^{\mathrm{I}} \mathrm{A}=\mathrm{CH}_{2}$
$(\alpha)_{1}^{20}=-3^{8} \pm 2^{\circ}$
$\frac{\mathrm{R}}{-\mathrm{CH}_{3}{ }^{2} \mathrm{CO}-\mathrm{A}=\mathrm{CH}_{2}}$
$\frac{\mathrm{R}=\mathrm{CH}^{3}{ }^{3} \mathrm{CO}^{-} \mathrm{A}=\mathrm{CO}}{\mathrm{F} .122-\mathrm{I} 26^{\circ}}$
$\mathrm{R}_{f}=0,23$ (Fig. 15$)$
$(\alpha)_{1}^{20}=-46 \pm 2^{\circ}$
$R_{t}=0,61$
$\mathrm{R}_{f}=0,52$ (Fig. 15) 
Il était donc certain que les larves de reines ne se développaient pas en absence de stérols. De plus, il devenait nécessaire de vérifier la présence de stérols dans la gelée royale.

B) Isolement du méthylène-24 cholestérol à partir de la gelée royale

Nous avons recherché la présence de stérols sur un échantillon de gelée royale (correspondant à environ $3 \mathrm{~g}$ de matière sèche).

Après une série d'extractions par le mélange éther-chloroforme $3: 1$, nous avons obtenu $374 \mathrm{mg}$ de produit, qui ont été ensuite directement chromatographiés sur colonne d'acide silicique.

Les éluats par le mélange benzène-éther $95: 5$, soit $9 \mathrm{mg}$, présentent seuls une réaction de Liebermann positive, et montrent, par chromatoplaque, une tache de même $\mathrm{R}_{f}(0,25)$ que le méthylène-24 cholestérol authentique.

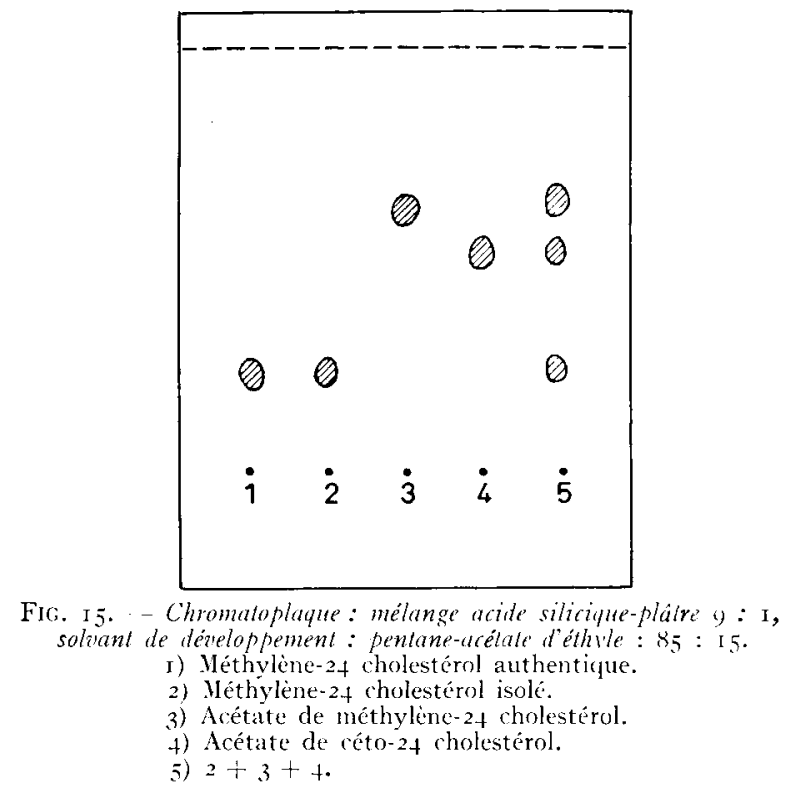

Après cristallisation dans le méthanol, nous avons obtenu $3 \mathrm{mg}$ de plaques incolores $\mathrm{F}$. $\mathrm{I}_{3} 8-\mathrm{I}_{4} \mathrm{I}^{\circ}$.

Le spectre IR-de cette substance est identique à celui du méthylène-24 cholestérol authentique, et présente bien les bandes à 6 , I et I I,3 $\mu$ caractéristiques du groupe méthylènique. Le point de fusion de mélange avec la substance authentique ne présente pas de dépression $\mathrm{F}$. I38-I4 $\mathrm{I}^{\circ}$.

\section{C) Conclusions}

La présence de méthylène-24 cholestérol dans la gelée royale règle le problème des stérols alimentaires des larves royales. De même que dans le pollen, il existe dans la gelée royale d'autres stérols, dont la séparation et l'étude sont difficiles. 
TABLEAU 5

Constantes physiques du méthylène-24 cholestérol

\begin{tabular}{|c|c|c|c|}
\hline \multirow{2}{*}{ Méthylène-2's cholestérol isolé de: } & \multicolumn{2}{|c|}{ Constantes physiques } & \multirow{2}{*}{$\mathrm{R}_{j}$ chromatoplaques } \\
\hline & I: & $(\alpha)_{1}-\mathrm{CHCl}_{3}$ & \\
\hline Huîtres $\ldots \ldots \ldots \ldots \ldots \ldots \ldots$ & $143^{\circ}$ & $-3^{\prime}, 8^{\circ}$ & \\
\hline Reines d'abeilles $\ldots \ldots \ldots \ldots \ldots$. & $138-145^{\circ}$ & $-31,6 \pm 6^{\circ}$ & $0,24 \pm 5 \%$ \\
\hline Abeilles ouvrières $\ldots \ldots \ldots \ldots$ & $138-140^{\circ}$ & $-31,6 \pm 2^{\circ}$ & $0,24 \pm 5 \%$ \\
\hline Pollen $\ldots \ldots \ldots \ldots \ldots \ldots \ldots$ & $141-145^{\circ}$ & $-29 \pm 1^{\circ}$ & $0,24 \pm 5 \%$ \\
\hline Larves royales ......... & $140-143^{\circ}$ & $-38 \pm 2^{\circ}$ & $0,23 \pm 5 \%$ \\
\hline Gelée royale $\ldots \ldots \ldots \ldots \ldots$ & $138-111^{\circ}$ & & $0,25 \pm 5 \%$ \\
\hline
\end{tabular}

\section{D) Partie expérimentale}

\section{a) Isolement à partir des larves royales}

$600 \mathrm{mg}$ de la fraction neutre provenant de l'extraction générale des graisses (chapitre I) ont été chromatographiés sur une colonne de $200 \mathrm{~g}$ d'acide silicique Mallinckrodt. Les élutions sont de $200 \mathrm{ml}$.

fractions
I à 3
4 à 6
7 à 9
I0 ̀̇ I I
I 2 à 13
I4 à I 6
I7 à 20
21 à 23

solvants
benzène
benzène-éther $98: 2$
benzène-éther $95: 5$
benzène-éther $95: 5$
benzène-éther $95: 5$
benzène-éther $85: 15$
benzène-éther I $: 1$
éther

mg
I $2 \mathrm{I} \mathrm{mg}$
I $2 \mathrm{mg}$
I0I $\mathrm{mg}$
$206 \mathrm{mg}$
$86 \mathrm{mg}$
$57 \mathrm{mg}$
traces
traces

Liebermann

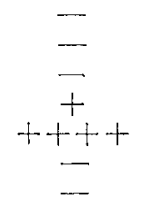

La réaction de Liebermann n'est fortement positive que pour les fractions Io et I I éluées au mélange benzène-éther 95: 5 ; ces fractions correspondent à $206 \mathrm{mg}$ de produit, qui, après deux cristallisations dans le méthanol, donnent $45 \mathrm{mg}$ de plaques incolores $\mathrm{F}$. $144^{\circ-143^{\circ}}$.

Ces cristaux ont été étudiés par chromatoplaques, dans le système pentane-acétate d'éthyle 85 : I 5 . Après migration du solvant de développement, et séchage de la plaque, on révèle en pulvérisant une solution de chlorure d'antimoine ( 33 p. I00 dans le chloroforme) et en chauffant à $200^{\circ}$ jusqu'à apparition des taches.

L'analyse élémentaire conduit à la formule $: \mathrm{C}_{28} \mathrm{H}_{46} \mathrm{O}$

$\begin{array}{llrrr}\text { Trouvé } & \mathrm{C} \% & 84,35 & \mathrm{H} \% & \text { I I,75 } \\ \text { Calculé } & & 84,35 & & \text { I } 1,63\end{array}$

\section{Formation d'un acétate.}

I $2 \mathrm{mg}$ de cristaux ont été dissous dans I ml de pyridine anhydre, et additionnés de o,9 ml d'anhydride acétique.

Après 72 heures à la température ambiante, on amène à sec, en entraînant la pyridine par additions successives de $0,5 \mathrm{ml}$ de benzène.

On reprend ensuite par Io $\mathrm{ml}$ d'éther; on lave 2 fois par $3 \mathrm{ml}$ d'acide chlorhydrique $\mathrm{N}, 2$ fois par $3 \mathrm{ml}$ d'eau, puis 2 fois par $3 \mathrm{ml}$ de carbonate de sodium $\mathrm{N}$ et enfin 2 fois par $3 \mathrm{ml}$ d'eau. On sèche la couche éthérée sur sulfate de sodium, et on obtient, après évaporation, i I mg d'acétate brut, qui, après cristallisation dans le méthanol, fournissent $8 \mathrm{mg}$ de plaques incolores. 


\section{Formation d'un acétate de céto-24 cholestérol.}

$50 \mathrm{mg}$ d'acétate de méthylène-24 cholestérol ont été dissous dans $10 \mathrm{ml}$ d'acide acétique. Après avoir dosé le débit d'ozone à l'aide d'une solution titrée d'hyposulfite, on fait passer, dans la solution acétique de l'acétate de méthylène-24 cholestérol, ıo fois la quantité théorique d'ozone.

On ajoute ensuite $12 \mathrm{ml}$ d'eau, et on porte à ébullition $5 \mathrm{mn}$. On extrait ensuite 3 fois par $15 \mathrm{ml}$ de chloroforme, on lave les solutions chlofoformiques à l'eau, on les sèche, et on évapore le chloroforme. On obtient ainsi $52 \mathrm{mg}$ de produit. Ces $52 \mathrm{mg}$ sont ensuite chromatographiés sur une colonne de $3 \mathrm{~g}$ d'acide silicique.

fractions
I à 5
6 à 8
9 à I1
I 2 à 14
I 5 à 17
I 8 à 20
2 I à 23

$\begin{array}{lr}\text { éluant } & \mathrm{mg} \\ \text { benzène } & 33 \mathrm{mg} \\ \text { benzène-éther } 99: \mathrm{I} & 2 \mathrm{mg} \\ \text { benzène-éther } 98: 2 & \text { traces } \\ \text { benzène-éther } 95: 5 & \mathrm{I} \mathrm{mg} \\ \text { benzène-éther } 9: \mathrm{I} & 7 \mathrm{mg} \\ \text { benzène-éther } 7: 3 & \text { traces } \\ \text { benzène-éther } \mathrm{I}: \mathrm{I} & 3 \mathrm{mg}\end{array}$

La fraction contenant l'acétate de céto-24 cholestérol est la fraction I 5 à i 7 .

Les acétates de méthylène-24 cholestérol et de céto-24 cholestérol ont été étudiés par chromato-plaques en révélant :

- avec une solution de chlorure d'antimoine ; cholestérol.

- avec une solution de dinitro-2,4 phényl hydrazine, à saturation, pour l'acétate de céto-24

\section{b) Isolement à partir de la gelée royale}

10,7 g de gelée royale ont été desséchés sous vide. On obtient $3 \mathrm{~g}$ environ d'une substance solide blanche, contenant encore un peu d'eau. Nous avons extrait ces $3 \mathrm{~g}$ par $250 \mathrm{ml}$ de chloroforme, une heure à reflux.

La couche chloroformique, séparée du résidu, a été lavée, puis séchée sur sulfate de sodium, et amenée à sec. On obtient $374 \mathrm{mg}$ de produit. Ces $374 \mathrm{mg}$ ont été chromatographiés sur I $5 \mathrm{~g}$ d'acide silicique.

\begin{tabular}{|c|c|c|c|}
\hline fractions & éluant & $\mathrm{mg}$ & Liebermann \\
\hline-- & - & — & - \\
\hline I à 4 & benzène & $8 \mathrm{mg}$ & 一 \\
\hline 5 à 6 & benzène-éther $9:$ I & $9 \mathrm{mg}$ & $t+t$ \\
\hline 7 à 9 & benzène-éther $9:$ I & $208 \mathrm{mg}$ & 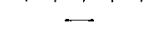 \\
\hline IOà I 2 & benzène-éther $8: 2$ & $93 \mathrm{mg}$ & - \\
\hline I3à I 5 & benzène-éther I : I & $20 \mathrm{mg}$ & - \\
\hline 16 à 20 & éther & traces & \\
\hline
\end{tabular}

Les fractions 5 et 6 cristallisées dans le méthanol, fournissent $3 \mathrm{mg}$ de plaques incolores F. I 38 $\mathbf{I}_{4} \mathbf{I}^{\mathrm{O}}$; le point de fusion de mélange avec un méthylène-24 cholestérol authentique ne présente pas de dépression. De la même manière que pour les larves royales, nous avons testé ces cristaux par chromatoplaques.

Le spectre IR est identique à celui de la substance authentique.

\section{CONCLUSIONS}

Les recherches décrites dans ce travail montrent la présence dans les larves de reines d'abeilles, d'un mélange d'esters de l'acide hydroxy-Io décène-2 trans oïque, et il semble que le premier stade de la transformation biologique de cet acide soit son estérification. Dans cette même fraction l'existence de polymères de l'acide hydroxy-Io décène-2 trans oïque est vraisemblable. L'importance de ce point est soulignée par une hypothèse émise par Sir Robert RoBINSON (I960), concernant l'activité anti-leucémique de cet acide et des estolides pouvant en dériver. 
La gelée royale et les larves de reines contiennent des acides dicarboxyliques (adipique, pimélique, subérique) dont la présence n’avait pas encore été signalée. Ces acides pourraient participer à 1'activité antileucémique attribuée à la gelée royale.

Dans une autre série de recherches sur la composition de la gelée royale, le méthylène-24 cholestérol a pu être isolé et identifié. C'est la première fois que ce stérol est signalé dans cette sécrétion. Les abeilles ouvrières sécrètent ce stérol à l'état libre, et apportent ainsi des stérols aux jeunes larves de reines, uniquement nourries de gelée royale. Ce stérol est aussi présent dans les lipides de larves de reines.

A l'encontre de la gelée royale qui possède une activité antibiotique très nette, les larves de reines sont dénuées de cette activité. Ce fait est peut-être dû à l'absence de quantités importantes d'acide hydroxy-Io décène- 2 trans oïque libre, dans les larves.

Ces recherches apportent donc des précisions nouvelles sur la composition de la gelée royale, et des larves reines.

Ręu pour publication en septembre 1962

\section{REMERCIEMENTS}

Ce travail a été effectué à l'Institut de Chimie des Substances naturelles, dirigé par M. le Professeur E. LEDERER. Je suis heureuse de lui exprimer ma respectueuse reconnaissance pour l'intérêt avec lequel il a suivi ces recherches.

Ce travail a été réalisé sous la direction de M. BARbier, Maître de recherches au C. N. R. S. Je tiens particulièrement à le remercier pour ses conseils éclairés et pour l'aide constante qu'il m'a toujours apportée.

J'adresse mes plus vifs remerciements à M. le Professeur Dufrenoy pour ses nombreux encouragements.

Je tiens enfin à remercier M. Chauvin et M. Louveaux pour l'intérêt porté à ce travail, et pour le matériel fourni.

\section{SUMMARY}

SOME COMPOUNDS IN TUE ROYAL JELLY AND LARVAE OF QUEEN BEES

This work describes a study of the lipids present in the royal jelly and in the larvae of the queens.

The principal new compounds isolated and identified were:

- esters of ro-hydroxy 2-decenoic acid with myristic, palmitic, stearic and sebaric acids (royal larvae) ;

— dicarboxylic, adipic, pimelic and suberic acids (larvae and royal jelly);

- finally, methylene-24 cholesterol (larvae and royal jelly).

\section{RÉFÉRENCES BIBLIOGRAPHIQUES}

Barier M., Reichetein T., Schindler O., I.kiderer li., 195\%. Isolation of 24-Methylene Cholesterol from Honey Bees (.tpis mellifica L..). Natme, 184. 732-734.

Barbier M., Schininer 0 ., 1959. Isolierun!r von 24-Methylen-cholesterin aus Königinnen und Arbeiterimnen der Honigbiene (Apis mellifica L.). Ilelu. Chim. Acla, 42, 1998-2005.

Barbier M.. Lenerer E., igho. Structure chimique de la "substance royale "de la reine d'abeille (Ipis mellifica L.). C. R. Acat. Sci., 250, $44^{67-4+609 .}$ 
Barbier M., Lederer l., Nomura T., I960. Synthèse de l'acide ceto-g décéne-2 trans oïque (Substance royale) et de l'acide céto-8 nonène-2 trans oique. C. R. Acad. Sci., 251, II 33-I I 35 .

Barbier M., Huget M. F., Lederer E., i960. Isolement du 24-méthylène cholestérol à partir du pollen de différentes plantes. Bull. Soc. Chim. Biol., 42, 9r-97.

Barbier M., PAIN J., I 960. Étude de la sécrétion des glandes mandibulaires des reines et des ouvrières d'abeilles (Apis mellifica L.) par chromatographie en phase gazeuse. C. R. Acad. Sci., 250, 3740-3742.

Barker S. A., Foster A. B., Lamb D. C., r959. Identification of ro Hydroxy $\Delta^{2}$ decenoic acid in royal jelly. Nature, 183, $996-997$.

Benton F. L., Kiess A. A., I960. A preparation of ro-hydroxy-decanoid acid. J. Org. Chem., 25, $270-27$ I. Blum M. S., Novak A. F., Taber S., I959. Io-hydroxy- $\Delta^{2}$ decenoic acid, an antibiotic found in Royal Jelly. Science, 130, $45^{2-453}$.

Brown W. H., Freure R. J., I959. Some carboxylic acids present in Royal Jelly. Can. J. Chem., 37, 20422046.

Brown W. H., Fetader E. E., ig6r. A new fatty acid from Royal Jelly. Nalure, 190, 88.

Brown W. H., Felacer E. E., Freure R. J., i96i. Some new components of Royal Jelly. Canad. J. Chem., 39, $1086-1089$.

Butenand A., Rembold H., i957. Über der Wiezelzellerfuttersaft der Honigbiene Hoppe-Seyl. Z., 308, $284-289$.

Butenandt A., REubold H., I958. The Royal Jelly of the honey bee. Isolation of 2-amino 4-hydroxy-6-(I,2dihydroxypropyl) pteridin. Z. Physiol. Chem., 311, 79-83.

ButenandT A., 1959. Wirkstoffe des Insektenreiches. Natumissenschaiten, 15, 46I-471.

ButLeR C. G., 1957. The control of ovary development in worker honey bee (Apis mellifica L). Experientia, 13, 256 .

Butler C. G., Callow R. K., Johnston N. C., 1959. Extraction and purification of "queen substance " of honey bees. Bee world, 41, (6), I 52-153.

Chauvin R., i959. La valeur diététique et thérapeutique des produits de la ruche. Miels, Pollans, Gelée royale. Produits pharmaceutiques, 14 I-27.

Clark A. J., Bloch K., I959. The absence of sterol syntnesis in insects. J. Biol. Chem., 231, 2578-81.

Clark A. J., Bloch K., I959. Function of sterols in Dermestes vulpinus. J. Biol. Chem., 234, 2583-88.

ClarK A. J., Bloci K., 1959. Conversion of ergosterol to 22-dehydrocholesterol in Blattella germanicum. J. Biol. Chem., 234, $2589-94$.

ENGLish J., I94I. The synthesis of some analogs of traumatic acid. J. Amer. Chem. Soc., 63, $941-943$.

Forrest I. S., Mitciell H. K., I955. Pteridines from Drosopinila. Isolation and identification of three more pteridines. J. Amer. Chem. Soc., 77, 4865-69.

Fray G. I., JAEger R. H., Robinson R., i960. Synthesis of cis-io-hydroxydec-2-enoic acid. Tetrahedron letters, 13, 34-35.

Gardner T.S., I948. Pantothenic acid as a longevity factor in Royal Jelly. J. Gerontol, 3, 1-8.

Idter D. R., FAGERLund U. H. M., I955. Marine sterols. Isolation of 24 methylene cholesterol from molluscs. J. Amer. Chem. Soc., "77, 4I $42-44$.

Konicek E., Levinson Z. H., I960. Metabolism of $\beta$ sitosterol and other lipids in the presence of acetate $2^{-14} \mathrm{C}$ by blowfly larvae. Nature, 188, I023-25.

Lipke H., Fraenkel G., I956. Insect nutrition. Ann. Rev. Entonology, 1, 17-44.

Monroe R. E., ig6o. Effect of dietary cholesterol on house fly reproduction. 53, 821-824.

Morgan J. F., Tolnai S., Townsend G. F., r96o. In vitro antitumor activity of fatty acids. Saturated dicarboxylic acids. Canad. J. Biol. Physiol., 38, 597-603.

PAIN J., 1955. Influence des reines mortes sur le développement ovarien des jeunes ouvrières d'abeilles. Insectes sociaux, $2,35 \cdot 43$.

Patterson E. L., Broquist H. P., Albrecirt A. M., Saltza M. II., Stokstad E. L. R., i955. A new pteridine in urine required for the growth of the protozoon Crithidia facasciculata. J. Amer. Chem. Soc., ryry, $3167-3168$.

Patterson E. L., Milstrey R., Stokstad E. L. R., i956. The synthesis of a pteridine required for the growth of Crithidia fasciculata. J. Amer. Chem. Soc., 78, 4I 5-4I7.

Prarson P. B., Burgin C. J., r94i. The pantothenic acid content of Royal Jelly. Proc. Soc. Exp. Biol. Med., 48, 4I5-4I7.

RuErn W. W., 1933. Uber die Entstehung des weiblichen Dimorphismus im Bienenstaats. Arch. Entro. Mech. Org., 129, 60I-665.

Robinson sir Robert, I960. The biological activity and synthesis of Royal Jelly Acid. Croatica chemica Acta., 32, II9-1 22.

Simpson J., 1960. The functions of the salivary glands of Apis mellifera. J. Insect Physiol, 4, 107-122. 'Townsend G. F., Lucas C. C.y I940. The chemical nature of Royal Jelly. Acid. Biochem. J., 34, I155-II62. Townsend G. F., MoRgan J. F., Hazlett B., I959. Activity of Io-hydroxydecenoic acid from Royal Jelly against experimental leukaemia and ascitics tumors. Nature, 183, I 270-I 27 r. 
Townend G. F., Morgan J. If., Tolna S., Hazlett B., Morto II. J., Schlel R. W., ig6o. Studies on the in vitro antitumor activity of fatty acids-10-hydroxy-2 decenoic acid from Royal Jelly. Cancer Res., 20, 503-510.

Viscontini M., Loeser F., Karrer P., Hadorn E., 1955. Fluoreszierende stoffe aus Drosophila melanogaster. Helv. Chem. Acta., 38, I $222-\mathrm{r} 224$.

Viscontini M., Scimid H., Hadorn E., i955. Isolierung fluoreszierender Stoffe aus Astacus fluviatilis. Experientia., 11, 390-392.

Viscontini M., Kehn A., Egeliaaf A., 1956. Isolierung floreszierender Stoffe aus Ephetia kükuiella. Z. Xaturforsch, $11 b, 50 \mathrm{I}-504$.

Zaffarowi A., Burton R. B., Keutuann E. H., 1950. Adrenal cortical homones : analysis by paper partition chromatography and occurence in the urine of nomal persons. Science, 111, 6-8. 